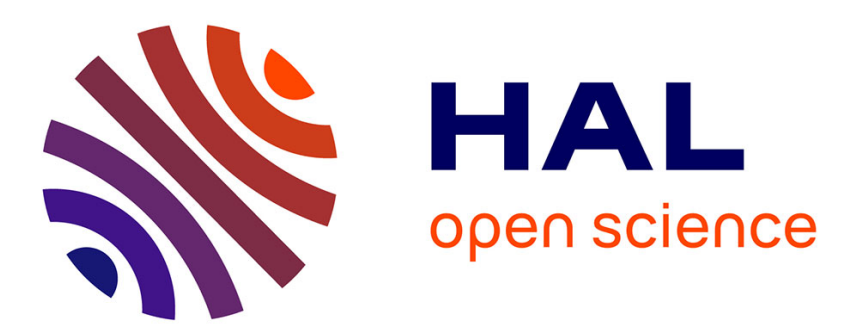

\title{
Centered-potential regularization for the advection upstream splitting method
}

Martin Parisot, Jean-Paul Vila

\section{To cite this version:}

Martin Parisot, Jean-Paul Vila. Centered-potential regularization for the advection upstream splitting method . SIAM Journal on Numerical Analysis, 2016. hal-01152395v2

\section{HAL Id: hal-01152395 \\ https://inria.hal.science/hal-01152395v2}

Submitted on 21 Sep 2016

HAL is a multi-disciplinary open access archive for the deposit and dissemination of scientific research documents, whether they are published or not. The documents may come from teaching and research institutions in France or abroad, or from public or private research centers.
L'archive ouverte pluridisciplinaire HAL, est destinée au dépôt et à la diffusion de documents scientifiques de niveau recherche, publiés ou non, émanant des établissements d'enseignement et de recherche français ou étrangers, des laboratoires publics ou privés. 


\title{
CENTERED-POTENTIAL REGULARIZATION FOR ADVECTION UPSTREAM SPLITTING METHOD *
}

\author{
MARTIN PARISOT ${ }^{\dagger}$ AND JEAN-PAUL VILA P $^{\ddagger}$
}

\begin{abstract}
The current paper is devoted to a centered IMEX scheme in multi-dimensional framework for a wide class of multicomponent and isentropic flows. The proposed strategy is based on a regularized model where the advection velocity is modified by the gradient of the potential of the conservative forces in both mass and momentum equations. The stability of the scheme is ensured by the dissipation of mechanic energy, which stands for a mathematical entropy, under an advective CFL condition. The main physical properties such as positivity, conservation of the total momentum and conservation of the steady state at rest are satisfied. In addition, asymptotic preserving properties in the regimes ('incompressible' and 'acoustic') are analyzed. Finally, several simulations are presented to illustrate our results in a simplified context of oceanic flows in one dimension.
\end{abstract}

Key words. conservation laws, low-Mach number, low-Froude number, well-balanced scheme, entropy dissipation, asymptotic preserving

AMS subject classifications. 35L60, 76M12, 86A05, 76E20, 35B40

\section{DOI. XXXXX}

1. Introduction. In the current paper, we are interested in a system of conservation laws with a large number $L$ of unknowns $\rho_{i}(t, X) \in \mathbb{R}_{+} /\{0\}$ satisfying an advection equation with their own velocity $U_{i}(t, X) \in \mathbb{R}^{d}$ in a multidimensional framwork $d \in \mathbb{N} \backslash\{0\}$, where $X \in \Omega \subset \mathbb{R}^{d}$ is the space variable and $t \geq 0$ is the time variable. The space domain is assumed to be periodic and its measure denoted by $|\Omega|$ is finite. The force field applied to the fluids is supposed to be irrotational, defined by the scalar potential $\phi_{i}(\boldsymbol{\rho}, X) \in \mathbb{R}$ with $\boldsymbol{\rho}=\left(\rho_{1}, \ldots, \rho_{L}\right)^{\top}$. The advection model reads

$$
\left\{\begin{array}{l}
\partial_{t} \rho_{i}+\nabla \cdot\left(\rho_{i} U_{i}\right)=0 \\
\partial_{t}\left(\rho_{i} U_{i}\right)+\nabla \cdot\left(\rho_{i} U_{i} \otimes U_{i}\right)=-\rho_{i} \nabla\left(\frac{\phi_{i}}{\varepsilon^{2}}\right)
\end{array}\right.
$$

with a given initial condition $\rho_{i}(0, X)=\rho_{i}^{0}(X)>0$ and $U_{i}(0, X)=U_{i}^{0}(X)$. In the following, we refer to the scalar unknowns $\rho_{i}$ as the masses and to the second equation of $\left(M_{\varepsilon}^{t}\right)$ as the momentum balance. The parameter $\varepsilon$ is the dimensionless number characterizing the ratio of the inertial and potential forces. In addition, the potential energy $\mathcal{E}(\boldsymbol{\rho}, X)$ is defined by $\partial_{\rho_{i}} \mathcal{E}:=\phi_{i}$, the kinetic energy by $\mathcal{K}_{i}:=\frac{\rho_{i}}{2}\left\|U_{i}\right\|^{2}$ and the mechanical energy of the model by $E:=\frac{\mathcal{E}}{\varepsilon^{2}}+\sum_{i=1}^{L} \mathcal{K}_{i}$. In order to lead to physical solutions, for any positive mass $\rho_{i}>0$, the potential energy should be a strictly convex function of the masses. More precisely, the Hessian $\mathbf{H}(\boldsymbol{\rho}, X)$ of the potential energy $\mathcal{E}$, defined by $\mathbf{H}_{i j}=\partial_{\rho_{j} \rho_{i}}^{2} \mathcal{E}=\partial_{\rho_{j}} \phi_{i}$ has to be positive-definite and the model

\footnotetext{
${ }^{*}$ Received by the editors XXXXX; accepted for publication (in revised form) XXXXX; published electronically XXXX.

http://www.siam.org/journals/sinum/XXXXX.html

$\dagger$ INRIA Paris, ANGE Project-Team, 75589 Paris Cedex 12, France; CEREMA, ANGE ProjectTeam, F-60280 Margny-Lès-Compiègne, France; LJLL, UPMC Université Paris VI, Sorbonne Universités, UMR CNRS 7958, F-75005 Paris, France; supported by the SHOM grant 15CR02 (martin.parisot@inria.fr)

${ }^{\ddagger}$ Institut de Mathématiques de Toulouse, UMR CNRS 5219, INSA, F-31077 Toulouse, France (vila@insa-toulouse.fr)
} 
$\left(M_{\varepsilon}^{t}\right)$ should satisfy the following entropy inequality, corresponding to the second law of thermodynamics

$$
\partial_{t} E+\sum_{i=1}^{L} \nabla \cdot\left(\left(\rho_{i} \frac{\phi_{i}}{\varepsilon^{2}}+\mathcal{K}_{i}\right) U_{i}\right) \leq 0 .
$$

The solution of $\left(M_{\varepsilon}^{t}\right)$ satisfies many steady states, which are generally hard to estimate. As is usual in the literature [2, 4, 17, 34, 38], only the steady state at rest is considered, i.e. with the additional condition $u_{i}=0$.

Proposition 1.1. The state defined by

$$
u_{i}=0 \quad \text { and } \quad \phi_{i}(\boldsymbol{\rho}(t, X), X)=\bar{\phi}_{i}
$$

is a steady state of the model $\left(M_{\varepsilon}^{t}\right)$, the so-called steady state at rest.

Note that several works present stability results for particular steady states with non-vanishing velocity, see for example [8, 22, 35].

In the current work, a particular attention is given to the resolution of the advection model $\left(M_{\varepsilon}^{t}\right)$ in the regime $\varepsilon \ll 1$. In such a regime, the main term of the potential is constant and is given by $\bar{\phi}_{i}=\frac{1}{|\Omega|} \int_{\Omega} \phi_{i}\left(\rho^{0}(X), X\right) \mathrm{d} X$ and the main term of the mass denoted by $\bar{\rho}_{i}(x)$ satisfies $\bar{\phi}_{i}=\phi_{i}(\overline{\boldsymbol{\rho}}(X), X)$. Two asymptotic models seem relevant, according to the time scale.

- For a short time scale $t=\underset{\varepsilon \rightarrow 0}{O}(\varepsilon)$, using the rescaled time $\tau=\frac{t}{\varepsilon}$ and assuming that the initial condition satisfies the scaling $U_{i}^{0}(X)=O(\varepsilon)$ and $\rho_{i}^{0}=\bar{\rho}_{i}+O(\varepsilon)$, the advection model $\left(M_{\varepsilon}^{t}\right)$ tends when $\varepsilon$ vanishes to a system of wave equations usually called acoustic regime defined by

$$
\begin{cases}\partial_{\tau} \widetilde{\phi}_{i}+\sum_{j=1}^{L} \overline{\mathbf{H}}_{i j} \nabla \cdot\left(\bar{\rho}_{j} \widetilde{U}_{j}\right) & =0 \\ \partial_{\tau} \widetilde{U}_{i}+\nabla \widetilde{\phi}_{i} & =0\end{cases}
$$

with the initial condition given by $\widetilde{\phi}_{i}(0, X)=\sum_{j=1}^{L} \overline{\mathbf{H}}_{i j}\left(\rho_{j}^{0}(X)-\bar{\rho}_{j}(X)\right)$ and $\widetilde{U}_{i}(0, X)=\frac{U_{i}^{0}(X)}{\varepsilon}$. The main part of the Hessian is given by $\overline{\mathbf{H}}_{i j}=\mathbf{H}_{i j}(\overline{\boldsymbol{\rho}}(X), X)$. For more details see [43].

- For a long time scale $t=\underset{\varepsilon \rightarrow 0}{O}(1)$, assuming that the Hessian of the potential energy is well-conditioned independently of $\varepsilon$, i.e. $\kappa=\underset{\varepsilon \rightarrow 0}{O}(1)$ with $\kappa$ the condition number of $\mathbf{H}$ and the initial condition satisfies the scaling $\rho_{i}^{0}=\bar{\rho}_{i}+O(\varepsilon)$ and $\nabla \cdot\left(\rho_{i}^{0} U_{i}^{0}\right)=$ $O(\varepsilon)$, the advection model $\left(M_{\varepsilon}^{t}\right)$ tends to a divergence free model usually called incompressible regime when $\varepsilon$ vanishes, i.e.

$$
\left\{\begin{aligned}
\nabla \cdot\left(\bar{\rho}_{i} \bar{U}_{i}\right) & =0 \\
\partial_{t} \bar{U}_{i}+\bar{U}_{i} \cdot \nabla \bar{U}_{i} & =-\nabla \psi_{i}
\end{aligned}\right.
$$

with the initial condition $\bar{U}_{i}(0, X)=U_{i}^{0}(X)$. The unknown $\psi_{i}(t, X)$ acts as a Lagrange multiplier to satisfy the divergence free condition. See $[23,24,28]$ for the case $L=1$. 
Proposition 1.2. Assume that the potential energy does not depend on the space variable, i.e. $\mathcal{E}(\boldsymbol{\rho}, X)=\mathcal{E}(\boldsymbol{\rho})$. Then the total momentum satisfies the following conservation law

$$
\partial_{t}\left(\sum_{i=1}^{L} \rho_{i} U_{i}\right)+\nabla \cdot\left(\sum_{i=1}^{L} \rho_{i} U_{i} \otimes U_{i}+\frac{P(\boldsymbol{\rho})}{\varepsilon^{2}} \mathrm{I}_{\mathrm{d}}\right)=0 .
$$

with $P(\boldsymbol{\rho})=\sum_{i=1}^{L} \rho_{i} \phi_{i}(\boldsymbol{\rho})-\mathcal{E}(\boldsymbol{\rho})$.

Many physical systems satisfy the assumptions of (1.1), Proposition 1.1 and the scaling of the Hessian $\kappa=\underset{\varepsilon \rightarrow 0}{O}$ (1). In the case of the one component system, i.e. $L=1$, the isentropic Euler equations [41, 44, 42, 15, 14] with or without gravity force can be written under the form $\left(M_{\varepsilon}^{t}\right)$. The isentropic pressure is a function of the mass and can be linked to the potential and the potential energy thanks to the relation $P=\rho \phi-\mathcal{E}$. In the case of polytropic fluids, where the pressure is given by $P=\lambda \rho^{\gamma}$ with $\lambda>0$ and the adiabatic coefficient $\gamma>1$, the potential energy, the potential and the Hessian are respectively given by

$$
\mathcal{E}=\lambda \frac{\rho^{\gamma}}{\gamma-1}+\rho g z, \quad \phi=\lambda \frac{\gamma \rho^{\gamma-1}}{\gamma-1}+g z \quad \text { and } \quad \mathbf{H}=\lambda \gamma \rho^{\gamma-2}
$$

where $z$ is the vertical coordinate. In this context, the dimensionless parameter $\varepsilon$ corresponds to the so-called Mach number. In the case without gravity force, i.e. $g=$ 0 , it is clear that the assumption of Proposition 1.2 is fulfilled. For a multicomponent system, i.e. $L>1$, the most obvious physical system which can be written under the form $\left(M_{\varepsilon}^{t}\right)$ is the mixture model [3]. Several numerical strategies can be cited to adapt the Riemann solvers to the non-conservative hyperbolic equations, see [6, 9, 32]. However, the eigenvalues, generally hard to approximate in the case of a large number of equations, are required for many numerical resolution of hyperbolic equations.

In the framework of free surface flow, the classical shallow water model [11] as well as the multilayer model $[1,27,36]$ can be written under the form $\left(M_{\varepsilon}^{t}\right)$. In this context, the so-called mass $\rho_{i}>0$ corresponds to the effective mass of the $i^{\text {th }}$ water column $\varrho_{i} h_{i}$ with $\varrho_{i}$ the density of the fluid and $h_{i}$ the layer thickness. The velocity $U_{i}$ corresponds to the mean horizontal velocity. In this context, the dimensionless parameter $\varepsilon$ corresponds to the so-called Froude number. Adopting the convention that the layers are numbered from the free surface to the bottom, the potential is given by

$$
\phi_{i}=g\left(B+\sum_{j=1}^{L} \frac{\varrho_{j} h_{j}}{\varrho_{\max (i, j)}}\right)
$$

with $B(X)$ the bottom elevation. The potential energy and the Hessian are respectively given by

$$
\mathcal{E}=g \sum_{i=1}^{L}\left(\left(B+\frac{1}{2} \sum_{j=1}^{L} \frac{\varrho_{j} h_{j}}{\varrho_{\max (i, j)}}\right) \varrho_{i} h_{i}\right), \quad \text { and } \quad \mathbf{H}_{i j}=\frac{g}{\varrho_{\max (i, j)}} .
$$

Note that the Hessian is positive-definite iff the layers are well-stratified, i.e. iff $\varrho_{i}<\varrho_{i+1}$. This assumption is a necessary (not sufficient) condition to ensure the 
multilayer shallow water model is hyperbolic, see [13, 30, 31]. Finally, in the case of flat topography, i.e. $B=0$, it is clear that the assumption of Proposition 1.2 is satisfied and leads in this context to the conservation of the momentum of the column of water.

Classical Riemann solvers are well-known to be to much dissipative in the asymptotic regime $\varepsilon \ll 1$, and require a very restrictive CFL condition, see [19]. More precisely, the asymptotic scheme obtained by passing $\varepsilon$ to zero in classical Riemann solvers is not consistent with the acoustic regime $\left(M_{0}^{\tau}\right)$, see [12]. The purpose of this work is to propose a numerical strategy $\left(S_{\varepsilon}^{\delta_{t}}\right)$ consistent with $\left(M_{\varepsilon}^{t}\right)$ for any value of $\varepsilon$ and satisfying the main physical properties (1.1), Proposition 1.2 and Proposition 1.1. In particular, the asymptotic scheme $\left(S_{0}^{\delta_{t}}\right)$ obtained by passing $\varepsilon$ to zero in the numerical scheme $\left(S_{\varepsilon}^{\delta_{t}}\right)$, is consistent with the incompressible regime $\left(M_{0}^{t}\right)$. Similarly, for small time step $\delta_{t}=\varepsilon \delta_{\tau}$, the asymptotic scheme $\left(S_{0}^{\delta_{\tau}}\right)$ obtained by passing $\varepsilon$ to zero in the numerical scheme $\left(S_{\varepsilon}^{\delta_{\tau}}\right)$, is consistent with the incompressible regime $\left(M_{0}^{\tau}\right)$. These properties, called in the literature asymptotic preserving (AP), see [21], can be illustrated by Fig. 1.

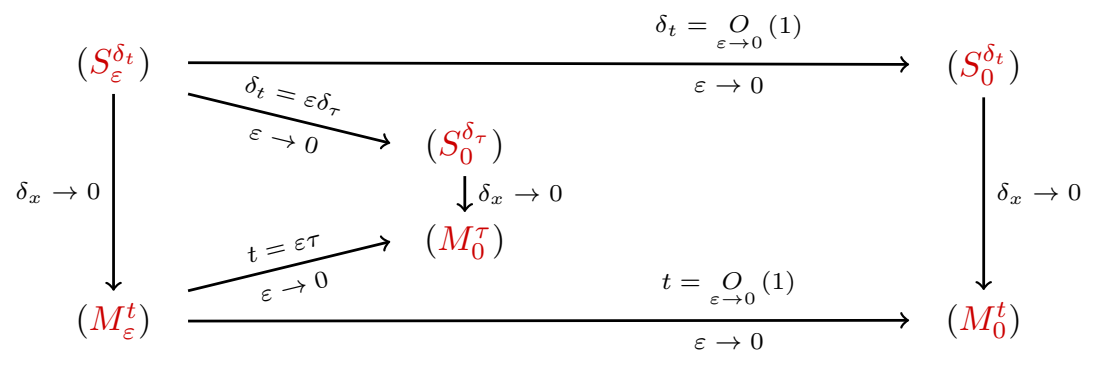

FIG. 1. Asymptotic preserving properties of the CPR scheme $\left(S_{\varepsilon}^{\delta_{t}}\right)$.

The numerical scheme studied in the current paper is a generalization of the scheme presented in [18]. It was applied for the discretization of the conservative two-fluid model with only one momentum equation, therefore the potential $\phi$ is a scalar. In addition, the potential $\phi$ is independent of the space variable. The main improvement of the current work is to propose a formulation able to deal with space dependent potentials and nonconservative products. Note that the space dependency of the potential is required in the modeling of source terms such as gravity in the isentropic Euler equation or the bathymetry in the shallow water model. The numerical strategy is described in a general multidimensional framework $\mathbb{R}^{d}$ and does not require a bound for the eigenvalues of the system. The stability of the scheme is ensured under an advective CFL condition, i.e. not restrictive for a continuous solution in the regime of small $\varepsilon$. The main results was already presented in [37] without mathematical proofs.

2. Centered-potential regularization of AUS method. In the current section, an adaptation of the so-called Advection Upstream Splitting Method (AUSM) introduced in [26] is proposed. The numerical strategy, from now on called CenteredPotential Regularization (CPR) is based on a centered estimate of the potential, as recommended in [12] to preserve the asymptotic limit, see Section 3. In addition, the centered estimate ensures the discrete stability of the steady state at rest, see Section 2.3. Then, the advection operator of the momentum equation is discretized 
using an upwind scheme according to the sign of the mass flux.

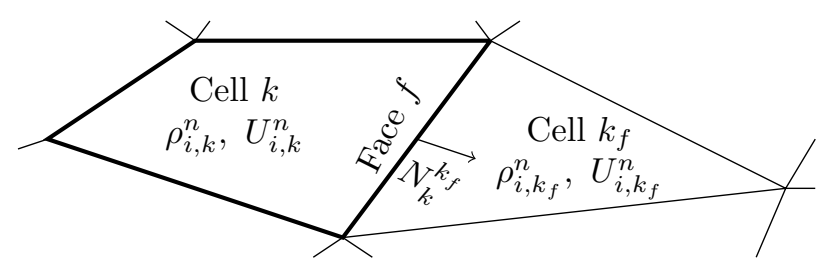

FIG. 2. Scheme of the numerical notations for $2 D$ case, $d=2$.

For any dimension $d \in \mathbb{N} \backslash\{0\}$, we consider a tessellation of $\Omega \subset \mathbb{R}^{d}$, denoted $\mathbb{T}$, composed of star-shaped control volumes. For any control volume $k \in \mathbb{T}$, its measure is denoted by $|k|$, its surface area by $|\partial k|$ and the set of its faces by $\mathbb{F}_{k}$. In addition, for any face $f \in \mathbb{F}_{k}$, its surface area is denoted by $|f|$, the neighbor of $k$ relative to the face $f$ by $k_{f} \in \mathbb{T}$ such that $k \cap k_{f}=f$ and the unit normal to the face $f$ outward to the control volume $k$ by $N_{k}^{k_{f}}$, see Fig. 2. The compactness of the control volume is denoted by $\ell_{k}=\frac{|k|}{|\partial k|}$ and the normalized face measure by $\mu_{f}^{k}=\frac{|f|}{|\partial k|}$. The numerical unknowns are approximated at time $t^{n+1}=t^{n}+\delta_{t}$ where $\delta_{t}$ is the time step at the $n^{\text {th }}$ iteration. The following numerical strategy is based on a cell-centered finite volume method, i.e. the numerical unknowns are the approximation of the averaged value of the mass $\rho_{i, k}^{n}>0$ and of the velocity $U_{i, k}^{n} \in \mathbb{R}^{d}$ at the time $t^{n}$ in each control volume $k \in \mathbb{T}$. The discretization of the potential is denoted by $\phi_{i, k}^{n}:=\phi_{i}\left(\boldsymbol{\rho}_{k}^{n}, X_{k}\right)$ and we use the following notations at the face: $2(a)_{f}:=a_{k}+a_{k_{f}}$ and $2[a]_{k}^{k_{f}}:=a_{k_{f}}-a_{k}$. The numerical scheme of the mass conservation $\left(M_{\varepsilon}^{t}\right)$ reads

$$
\rho_{i, k}^{n+1}=\rho_{i, k}^{n}-\frac{\delta_{t}}{\ell_{k}} \sum_{f \in \mathbb{F}_{k}} \mathcal{F}_{i, f}^{n+1} \cdot N_{k}^{k_{f}} \mu_{f}^{k}
$$

where the numerical mass flux $\mathcal{F}_{i, f}^{n+1}$ is an approximation of $\frac{1}{|f| \delta_{t}} \int_{t^{n}}^{t^{n+1}} \int_{f} \rho_{i} U_{i} \mathrm{~d} \sigma \mathrm{d} t$. Then, the momentum balance is approximated using an upwind scheme. It reads

$$
\begin{aligned}
\rho_{i, k}^{n+1} U_{i, k}^{n+1} & =\rho_{i, k}^{n} U_{i, k}^{n}-\rho_{i, k}^{n+1} \frac{\delta_{t}}{\ell_{k}} \sum_{f \in \mathbb{F}_{k}} \frac{\left(\phi_{i}^{n+1}\right)_{f}}{\varepsilon^{2}} N_{k}^{k_{f}} \mu_{f}^{k} \\
& -\frac{\delta_{t}}{\ell_{k}} \sum_{f \in \mathbb{F}_{k}}\left(U_{i, k}^{n}\left(\mathcal{F}_{i, f}^{n+1} \cdot N_{k}^{k_{f}}\right)^{+}-U_{i, k_{f}}^{n}\left(\mathcal{F}_{i, f}^{n+1} \cdot N_{k}^{k_{f}}\right)^{-}\right) \mu_{f}^{k}
\end{aligned}
$$

with the positive and the negative part functions defined by $2(\psi)^{ \pm}=|\psi| \pm \psi \geq 0$. The numerical mass flux is regularized using the centered potential variation, i.e.

$$
\left(S_{\varepsilon}^{\delta_{t}} . c\right) \quad \mathcal{F}_{i, f}^{n+1}:=\left(\rho_{i}^{n+1} U_{i}^{n}\right)_{f}-\gamma \delta_{t}\left(\frac{\rho_{i}^{n+1}}{\ell}\right)_{f} \frac{\left[\phi_{i}^{n+1}\right]_{k}^{k_{f}}}{\varepsilon^{2}} N_{k}^{k_{f}} .
$$

The regularization parameter $\gamma$ can be defined locally, at the face, as a function of the flow. The sensitivity of the solution to the numerical regularization parameter is clearly depending on the state variables. In the following, under the following implicit CFL condition

$$
\frac{\delta_{t}}{\ell_{k}} \sum_{f \in \mathbb{F}_{k}}\left|\mathcal{F}_{i, f}^{n+1} \cdot N_{k}^{k_{f}}\right| \mu_{f}^{k}<\rho_{i, k}^{n}
$$


and for $\gamma \geq 1$, an entropic stability result is highlighted, see Theorem 2.3. Note that the numerical scheme $\left(S_{\varepsilon}^{\delta_{t}}\right)$ is a nonlinear IMEX scheme. More precisely, the implicit CFL condition (2.1) can not be directly used to estimate a time step since it depends on the mass (through the potential) at the next time iteration. In addition, the mass conservation $\left(S_{\varepsilon}^{\delta_{t}} \cdot a\right)$ is a nonlinear coupled implicit scheme. One can use a Brouwer argument to show the existence of a fixed point. In practice, the scheme $\left(S_{\varepsilon}^{\delta_{t}} \cdot a\right)$ is solved using a Banach fixed point strategy and the time step is estimated using the previous approximation.

Proposition 2.1 (Positivity). Assume that the initial condition $\rho_{i, k}^{0}$ are positive. Then there exists a time step $\delta_{t}$ such that the CFL condition (2.1) is fulfilled and the mass approximations $\rho_{i, k}^{n}$ are positive.

Proof. Assume that the mass approximation at time iteration $n$ is positive, i.e. $\rho_{i, k}^{n}>0$, which is true for the initial condition. The CFL condition (2.1) is clearly satisfied for $\delta_{t}=0$ and it is not an equality case. Then since the mass approximations $\rho_{i, k}^{n+1}$ are continuous functions of the time step $\delta_{t}$, there exists a neighborhood of 0 for $\delta_{t}$ such that the condition is satisfied. Then by direct estimate, we have

$$
\rho_{i, k}^{n+1}=\rho_{i, k}^{n}-\frac{\delta_{t}}{\ell_{k}} \sum_{f \in \mathbb{F}_{k}} \mathcal{F}_{i, f}^{n+1} \cdot N_{k}^{k_{f}} \mu_{f}^{k}>2 \frac{\delta_{t}}{\ell_{k}} \sum_{f \in \mathbb{F}_{k}}\left(\mathcal{F}_{i, f}^{n+1} \cdot N_{k}^{k_{f}}\right)^{-} \mu_{f}^{k}
$$

which leads to the positivity of the mass approximations $\rho_{i, k}^{n+1}$.

To compare the implicit CFL condition (2.1) to a classical CFL condition, a more restrictive CFL condition but in the classical form is proposed. The numerical mass flux is bounded by

$$
\left(\mathcal{F}_{i, f}^{n+1} \cdot N_{f}^{k}\right)^{-} \leq\left|\left(\rho_{i}^{n+1} U_{i}^{n}\right)_{f} \cdot N_{k}^{k_{f}}\right|+\gamma \delta_{t}\left(\frac{\rho_{i}^{n+1}}{\ell}\right)_{f} \frac{\left|\left[\phi_{i}^{n+1}\right]_{k}^{k_{f}}\right|}{\varepsilon^{2}}
$$

Since the sum over the faces is normalized, the CFL condition can be computed by face and leads to a second order polynomial function $\left(V_{2} \frac{\delta_{t}}{\ell_{k}}\right)^{2}+V_{1} \frac{\delta_{t}}{\ell_{k}}-1$ which is negative when $\left(V_{1}+V_{2}\right) \frac{\delta_{t}}{\ell_{k}}<1$. After simplification, we get the following CFL condition

$$
\frac{\left|\left(\rho_{i}^{n+1} U_{i}^{n}\right)_{f} \cdot N_{k}^{k_{f}}\right|+\left(\rho_{i}^{n+1}\right)_{f} \sqrt{\frac{\gamma}{2} \frac{\left|\left[\phi_{i}^{n+1}\right]_{k}^{k_{f}}\right|}{\varepsilon^{2}}}}{\min \left(\rho_{i, k}^{n+1}, \rho_{i, k_{f}}^{n+1}\right)} \frac{\delta_{t}}{\min \left(\ell_{k}, \ell_{k_{f}}\right)}<\frac{1}{2}
$$

which is more restrictive than (2.1). Classical Godunov schemes are generally stable under a CFL condition of the form $\left(\left|U_{i}^{n} \cdot N_{f}^{k}\right|+\frac{c_{i}^{n}}{\varepsilon}\right) \frac{\delta_{t}}{\ell}<C s t$ see [16]. The celerity of the potential wave $\left(c_{i}\right)_{1 \leq i \leq L}$ corresponds to the square root of the eigenvalues of the matrix $\rho_{i} \mathbf{H}_{i j}$. In the limit $\varepsilon$ goes to zero, the wave potential is still large $c_{i}=\underset{\varepsilon \rightarrow 0}{O}(1)$ and the classical CFL condition becomes restrictive, i.e. $\frac{\delta_{t}}{\ell}=\underset{\varepsilon \rightarrow 0}{O}(\varepsilon)$. On the contrary, the main term of the potential is constant in space, i.e. $\phi_{i, k}^{n}=$ $\phi_{i, k_{f}}^{n}+\underset{\varepsilon \rightarrow 0}{O}\left(\varepsilon^{2}\right)$, see Section 3.2 and the CFL condition of the CPR scheme can be large, i.e. $\frac{\delta_{t}}{\ell}=\underset{\varepsilon \rightarrow 0}{O}(1)$. 
The regularization term in the numerical mass flux plays a role similar to the numerical viscosity introduced in the Lax-Wendroff scheme [25]. In particular, dispersive oscillations are present in the vicinity of the shock using the CPR scheme $\left(S_{\varepsilon}^{\delta_{t}}\right)$ as well as the Lax-Wendroff scheme. Similarly to the artificial viscosity (denoted by $B$ in [25]), the regularization parameter $\gamma$ can be increased in order to reduce the amplitude of these oscillations.

2.1. Consistency and accuracy order. In the current section, the consistency of the CPR scheme $\left(S_{\varepsilon}^{\delta_{t}}\right)$ in the sense of finite difference methods is analyzed. The compactness reads $\ell_{k}=\frac{|k|}{|\partial k|}=\frac{\delta_{x}}{2 d}$ with $d$ the dimension and $\delta_{x}$ is the standard space step definition, i.e. the distance between the center of two neighboring cells. For readability reasons, the space Taylor expansion is written in one direction only. The neighbors of the volume $k \in \mathbb{Z}$ in the considered direction are $k-1$ and $k+1$. The notation $k+1 / 2$ indicate a discretization at the face between the volume $k$ and $k+1$. Even if the index of the other directions are not indicated, the proof is established in multi-dimensional framework $d \in \mathbb{N} \backslash\{0\}$.

Proposition 2.2 (Consistency). Assume that the solution is smooth enough and the tessellation is a regular cartesian grid with a space step $\delta_{x}$. Then for any regularization coefficient $\gamma$ bounded with respect to $\delta_{t}$ and $\delta_{x}$ the CPR scheme $\left(S_{\varepsilon}^{\delta_{t}}\right)$ is consistent with the advection model $\left(M_{\varepsilon}^{t}\right)$. More precisely the modified system reads

$$
\left\{\begin{array}{r}
\partial_{t} \rho_{i}+\nabla \cdot\left(\rho_{i} W_{i}\right)=O\left(\delta_{t}^{2}, \delta_{x}^{2}, \frac{\delta_{t} \delta_{x}^{2}}{\varepsilon^{2}}\right) \\
\partial_{t}\left(\rho_{i} U_{i}\right)+\nabla \cdot\left(\rho_{i} U_{i} \otimes W_{i}\right)=-\rho_{i} \nabla\left(\frac{\phi_{i}}{\varepsilon^{2}}\right)-\frac{\delta_{t}}{2}\left(\nabla \cdot\left(\rho_{i} \partial_{t} U_{i} \otimes U_{i}\right)+\partial_{t}\left(\frac{\rho_{i}}{\varepsilon^{2}} \nabla \phi_{i}\right)\right) \\
+O\left(\delta_{t}^{2}, \delta_{x}, \frac{\delta_{t} \delta_{x}^{2}}{\varepsilon^{2}}\right) .
\end{array}\right.
$$

with the modified discharge $\rho_{i} W_{i}=\rho_{i} U_{i}-\frac{\delta_{t}}{2}\left(2 d \gamma \rho_{i} \nabla\left(\frac{\phi_{i}}{\varepsilon^{2}}\right)+\rho_{i} \partial_{t} U_{i}-U_{i} \partial_{t} \rho_{i}\right)$.

Proof. The exact solution of $\left(M_{\varepsilon}^{t}\right)$ at time $t^{n}$ and at the position $k \delta_{x}$ is denoted by $\left.\rho_{i}\right|_{k} ^{n}:=\rho_{i}\left(t^{n}, k \delta_{x}\right)$ and $\left.U_{i}\right|_{k} ^{n}:=U_{i}\left(t^{n}, k \delta_{x}\right)$. Let us focus firstly on the numerical mass flux $\left(S_{\varepsilon}^{\delta_{t}} \cdot c\right)$. The centered series leads to

$$
\begin{aligned}
\frac{1}{4}\left(\left.\rho_{i}\right|_{k+1} ^{n+1}+\left.\rho_{i}\right|_{k} ^{n+1}\right)\left(\left.\phi_{i}\right|_{k+1} ^{n+1}-\left.\phi_{i}\right|_{k} ^{n+1}\right) & =\left.\frac{\delta_{x}}{2} \rho_{i} \partial_{x} \phi_{i}\right|_{k+1 / 2} ^{n+1}+\left.\frac{\delta_{x}^{3}}{2} C\right|_{k+1 / 2} ^{n+1}+O\left(\delta_{x}^{5}\right) \\
& =\left.\frac{\delta_{x}}{2} \rho_{i} \partial_{x} \phi_{i}\right|_{k+1 / 2} ^{n+1 / 2}+\left.\frac{\delta_{x}^{3}}{2} C\right|_{k+1 / 2} ^{n+1 / 2}+O\left(\delta_{t} \delta_{x}, \delta_{x}^{5}\right)
\end{aligned}
$$

where $C=\frac{1}{8}\left(\partial_{x}^{2} \rho_{i} \partial_{x} \phi_{i}+\frac{\partial_{x}^{3} \phi_{i}}{3}\right)$ and

$$
\begin{aligned}
\frac{1}{2}\left(\left.\left.\rho_{i}\right|_{k+1} ^{n+1} U_{i}\right|_{k+1} ^{n}+\left.\left.\rho_{i}\right|_{k} ^{n+1} U_{i}\right|_{k} ^{n}\right) & \\
& =\left.\left.\rho_{i}\right|_{k+1 / 2} ^{n+1} U_{i}\right|_{k+1 / 2} ^{n}+\frac{\delta_{x}^{2}}{8} \partial_{x}\left(\left.\left.\rho_{i}\right|_{k+1 / 2} ^{n+1} U_{i}\right|_{k+1 / 2} ^{n}\right)+O\left(\delta_{x}^{4}\right) \\
& =\left.\rho_{i} U_{i}\right|_{k+1 / 2} ^{n+1 / 2}+\left.\frac{\delta_{t}}{2} T\right|_{k+1 / 2} ^{n+1 / 2}+\left.\delta_{x}^{2} D\right|_{k+1 / 2} ^{n+1 / 2}+O\left(\delta_{t}^{2}, \delta_{x}^{4}\right)
\end{aligned}
$$

with $D=\frac{1}{8} \partial_{x}\left(\rho_{i} U_{i}+\frac{\delta_{t}}{2} T\right)$ and $T=U_{i} \partial_{t} \rho_{i}-\rho_{i} \partial_{t} U_{i}$. Using a trapezoidal rule, the flux at time $t^{n}+\frac{\delta_{t}}{2}$ is a second order approximation of the integral of the flux along 
the time step. More precisely, we have

$$
\left.\mathcal{F}_{i}\right|_{k+1 / 2} ^{n+1}=\left.\left(\rho_{i} W_{i}+\delta_{x}^{2} D+\frac{\delta_{t} \delta_{x}^{2}}{\varepsilon^{2}} d \gamma C\right)\right|_{k+1 / 2} ^{n+1 / 2}+O\left(\delta_{t}^{2}, \delta_{x}^{4}\right)
$$

with $\left.\mathcal{F}_{i}\right|_{k+1 / 2} ^{n+1}$ the discrete flux $\left(S_{\varepsilon}^{\delta_{t}} . c\right)$ estimated with the exact solution. Using the centered series, the modified equation of the mass is obtained. Considering the momentum equation, the upwind scheme leads to the following series

$$
\begin{aligned}
\left.U_{i}\right|_{k} ^{n}\left(\left.\mathcal{F}_{i}\right|_{k+1 / 2} ^{n+1}\right. & \left.\cdot N_{k}^{k_{f}}\right)^{+}-\left.U_{i}\right|_{k_{f}} ^{n}\left(\left.\mathcal{F}_{i}\right|_{k+1 / 2} ^{n+1} \cdot N_{k}^{k_{f}}\right)^{-} \\
= & \left.\left(U_{i}+\frac{\delta_{t}}{2} \partial_{t} U_{i}\right) \rho_{i} W_{i}\right|_{k+1 / 2} ^{n+1 / 2}+O\left(\delta_{t}^{2}, \delta_{x}\right)
\end{aligned}
$$

The right-hand-side is finally written to get the modified momentum balance.

2.2. Stability and entropy dissipation. In the current section, a stability result based on the dissipation of the discrete mechanical energy, which stands for a mathematical entropy, is presented. Let us introduce the discrete kinetic energy $\mathcal{K}_{i, k}^{n}:=\frac{\rho_{i, k}^{n}}{2}\left\|U_{i, k}^{n}\right\|^{2}$, the discrete potential energy $\mathcal{E}_{k}^{n}:=\mathcal{E}\left(\boldsymbol{\rho}_{k}^{n}, X_{k}\right)$ and the discrete mechanical energy $E_{k}^{n}:=\frac{\mathcal{E}_{k}^{n}}{\varepsilon^{2}}+\sum_{i=1}^{L} \mathcal{K}_{i, k}^{n}$.

Theorem 2.3 (Dissipation of the discrete mechanical energy). Assume that the CFL condition (2.1) is fulfilled. Then for any regularization coefficient $\gamma \geq 1$, the discrete mechanical energy satisfies the following local entropy inequality

$$
E_{k}^{n+1} \leq E_{k}^{n}-\frac{\delta_{t}}{\ell_{k}} \sum_{f \in \mathbb{F}_{k}} \sum_{i=1}^{L}\left(\left(\mathcal{G}_{\mathcal{K}, i, f}^{n+1}+\frac{\mathcal{G}_{\mathcal{E}, i, f}^{n+1}}{\varepsilon^{2}}\right) \cdot N_{k}^{k_{f}}+\mathcal{H}_{\mathcal{K}, i, f}^{n+1}+\frac{\mathcal{H}_{\mathcal{E}, i, f}^{n+1}}{\varepsilon^{2}}\right) \mu_{f}^{k}
$$

with the fluxes $\mathcal{G}_{\mathcal{K}, i, f}^{n+1}$ and $\mathcal{H}_{\mathcal{K}, i, f}^{n+1}$ of kinetic energy defined by

$$
\begin{aligned}
\mathcal{G}_{\mathcal{K}, i, f}^{n+1} \cdot N_{k}^{k_{f}} & :=\frac{1}{2}\left\|U_{i, k}^{n}\right\|^{2}\left(\mathcal{F}_{i, f}^{n+1} \cdot N_{k}^{k_{f}}\right)^{+}-\frac{1}{2}\left\|U_{i, k_{f}}^{n}\right\|^{2}\left(\mathcal{F}_{i, f}^{n+1} \cdot N_{k}^{k_{f}}\right)^{-} \\
\text {and } \quad \mathcal{H}_{\mathcal{K}, i, f}^{n+1} & :=\delta_{t}\left[\frac{\rho_{i}^{n+1}}{\ell}\right]_{k}^{k_{f}}\left|\frac{\left[\phi_{i}^{n+1}\right]_{k}^{k_{f}}}{\varepsilon^{2}}\right|^{2}
\end{aligned}
$$

and the fluxes $\mathcal{G}_{\mathcal{E}, i, f}^{n+1}$ and $\mathcal{H}_{\mathcal{E}, i, f}^{n+1}$ of potential energy defined by

$$
\begin{aligned}
\mathcal{G}_{\mathcal{E}, i, f}^{n+1} \cdot N_{f}^{k} & :=\left(\phi_{i}^{n+1}\right)_{f} \mathcal{F}_{i, f}^{n+1} \cdot N_{k}^{k_{f}} \\
\text { and } \quad \mathcal{H}_{\mathcal{E}, i, f}^{n+1} & :=-\left[\phi_{i}^{n+1}\right]_{k}^{k_{f}}\left[\rho_{i}^{n+1} U_{i}^{n}\right]_{k}^{k_{f}} \cdot N_{k}^{k_{f}} .
\end{aligned}
$$

The proof of the theorem results from two lemmas that establish in the discrete framework the evolution of the kinetic energy and potential energy.

Lemma 2.4 (Kinetic energy). Assume that the CFL condition (2.1) is fulfilled. Then the discrete kinetic energy satisfies the following bound

$$
\mathcal{K}_{i, k}^{n+1} \leq \mathcal{K}_{i, k}^{n}-\frac{\delta_{t}}{\ell_{k}} \sum_{f \in \mathbb{F}_{k}}\left(\mathcal{G}_{\mathcal{K}, i, f}^{n+1} \cdot N_{f}^{k}+\mathcal{H}_{\mathcal{K}, i, f}^{n+1}-R_{\mathcal{K}, i, f}^{n+1}\right) \mu_{f}^{k}-\delta_{t} \frac{Q_{i, k}^{n+1}}{\varepsilon^{2}}
$$


where the fluxes of potential energy $\mathcal{G}_{\mathcal{K}, i, f}^{n}$ and $\mathcal{H}_{\mathcal{K}, i, f}^{n}$ are defined by (2.4), the source term and the discrete work of the forces are respectively given by

$$
R_{\mathcal{K}, i, f}^{n+1}:=\delta_{t}\left(\frac{\rho_{i}^{n+1}}{\ell}\right)_{f}\left|\frac{\left[\phi_{i}^{n+1}\right]_{k}^{k_{f}}}{\varepsilon^{2}}\right|^{2} \quad \text { and } \quad Q_{i, k}^{n+1}:=\frac{\rho_{i, k}^{n+1} U_{i, k}^{n}}{\ell_{k}} \cdot \sum_{f \in \mathbb{F}_{k}}\left[\phi_{i}^{n+1}\right]_{k}^{k_{f}} N_{k}^{k_{f}} \mu_{f}^{k}
$$

Proof. According to Proposition 2.1, the masses $\rho_{i, k}^{n+1}$ are positive. Then the scheme satisfied by the velocity $U_{i, k}^{n+1}$ is obtained by replacing the mass $\rho_{i, k}^{n}$ in the momentum scheme $\left(S_{\varepsilon}^{\delta_{t}} . b\right)$ using the mass scheme $\left(S_{\varepsilon}^{\delta_{t}} . a\right)$. More precisely

$$
U_{i, k}^{n+1}=U_{i, k}^{n}+\frac{\delta_{t}}{\ell_{k}} \sum_{f \in \mathbb{F}_{k}} \frac{U_{i, k_{f}}^{n}-U_{i, k}^{n}}{\rho_{i, k}^{n+1}}\left(\mathcal{F}_{i, f}^{n+1} \cdot N_{f}^{k}\right)^{-} \mu_{f}^{k}-\frac{\delta_{t}}{\ell_{k}} \sum_{f \in \mathbb{F}_{k}} \frac{\left(\phi_{i}^{n+1}\right)_{f}}{\varepsilon^{2}} N_{k}^{k_{f}} \mu_{f}^{k} .
$$

The centered-discretization of the potential at faces can replaced by the half-difference,

$$
\sum_{f \in \mathbb{F}_{k}}\left(\phi_{i}^{n+1}\right)_{f} N_{k}^{k_{f}} \mu_{f}^{k}=\sum_{f \in \mathbb{F}_{k}}\left[\phi_{i}^{n+1}\right]_{k}^{k_{f}} N_{k}^{k_{f}} \mu_{f}^{k}
$$

Using the equality $2 A \cdot(B-A)=\|B\|^{2}-\|A\|^{2}-\|B-A\|^{2}$ for any $A \in \mathbb{R}^{d}$ and $B \in \mathbb{R}^{d}$, the scalar product between the velocity scheme (2.8) and the $\rho_{i, k}^{n+1} U_{i, k}^{n}$ leads to

$$
\mathcal{K}_{i, k}^{n+1}=\mathcal{K}_{i, k}^{n}-\frac{\delta_{t}}{\ell_{k}} \sum_{f \in \mathbb{F}_{k}}\left(\mathcal{G}_{\mathcal{K}, i, f}^{n+1} \cdot N_{k}^{k_{f}}\right) \mu_{f}^{k}-\delta_{t} \frac{Q_{i, k}^{n+1}}{\varepsilon^{2}}+S_{i, k}^{n+1}
$$

where the source term $S_{i, k}^{n+1}$ coming from the numerical discretization is given by

$$
S_{i, k}^{n+1}:=\frac{1}{2} \rho_{i, k}^{n+1}\left\|U_{i, k}^{n+1}-U_{i, k}^{n}\right\|^{2}-2 \frac{\delta_{t}}{\ell_{k}} \sum_{f \in \mathbb{F}_{k}}\left\|\left[U_{i}^{n}\right]_{k}^{k_{f}}\right\|^{2}\left(\mathcal{F}_{i, f}^{n+1} \cdot N_{f}^{k}\right)^{-} \mu_{f}^{k} .
$$

The following step of the proof looks for an upper bound the source term $S_{i, k}^{n+1}$ in function of the potential variation. Using the velocity scheme (2.8) and Jensen's inequality, the advection term and the variation of potential are split, i.e.

$$
\begin{aligned}
S_{i, k}^{n+1} \leq & \rho_{i, k}^{n+1}\left(\frac{\delta_{t}}{\ell_{k}}\right)^{2} \sum_{f \in \mathbb{F}_{k}}\left|\frac{\left[\phi_{i}^{n+1}\right]_{k}^{k_{f}}}{\varepsilon^{2}}\right|^{2} \mu_{f}^{k} \\
& +\frac{1}{\rho_{i, k}^{n+1}}\left(\frac{\delta_{t}}{\ell_{k}}\right)^{2}\left\|\sum_{f \in \mathbb{F}_{k}}\left(\sqrt{\left(\mathcal{F}_{i, f}^{n+1} \cdot N_{f}^{k}\right)^{-}}\right)\left(2\left[U_{i}^{n}\right]_{k}^{k_{f}} \sqrt{\left(\mathcal{F}_{i, f}^{n+1} \cdot N_{f}^{k}\right)^{-}}\right) \mu_{f}^{k}\right\|^{2} \\
& -2 \frac{\delta_{t}}{\ell_{k}} \sum_{f \in \mathbb{F}_{k}}\left\|\left[U_{i}^{n}\right]_{k}^{k_{f}}\right\|^{2}\left(\mathcal{F}_{i, f}^{n+1} \cdot N_{f}^{k}\right)^{-} \mu_{f}^{k}
\end{aligned}
$$

Then the first term of the right-hand-side is split into an anti-symmetric part which leads to the flux $\mathcal{H}_{\mathcal{K}, i, f}^{n+1}$ given by (2.4) and the symmetric part which leads to a source term $R_{\mathcal{K}, i, f}^{n+1}$ estimated at the faces (2.7). Finally, using a Cauchy-Schwarz's inequality, 
the last terms of the right-hand-side is bounded by

$$
\begin{aligned}
S_{i, k}^{n+1} & \leq \frac{\delta_{t}}{\ell_{k}} \sum_{f \in \mathbb{F}_{k}}\left(R_{\mathcal{K}, i, f}^{n+1}-\mathcal{H}_{\mathcal{K}, i, f}^{n+1}\right) \mu_{f}^{k} \\
& +4 \frac{\delta_{t}}{\ell_{k}}\left(\sum_{f \in \mathbb{F}_{k}}\left\|\left[U_{i}^{n}\right]_{k}^{k_{f}}\right\|^{2}\left(\mathcal{F}_{i, f}^{n+1} \cdot N_{k}^{k_{f}}\right)^{-} \mu_{f}^{k}\right)\left(\frac{\delta_{t}}{\ell_{k}} \sum_{f \in \mathbb{F}_{k}} \frac{\left(\mathcal{F}_{i, f}^{n+1} \cdot N_{k}^{k_{f}}\right)^{-}}{\rho_{i, k}^{n+1}} \mu_{f}^{k}-\frac{1}{2}\right) .
\end{aligned}
$$

We conclude using the CFL condition (2.1).

Lemma 2.5 (Potential energy). The discrete potential energy satisfies the following bound

$$
\mathcal{E}_{k}^{n+1} \leq \mathcal{E}_{k}^{n}-\frac{\delta_{t}}{\ell_{k}} \sum_{f \in \mathbb{F}_{k}} \sum_{i=1}^{L}\left(\mathcal{G}_{\mathcal{E}, i, f}^{n+1} \cdot N_{f}^{k}+\mathcal{H}_{\mathcal{E}, i, f}^{n+1}-R_{\mathcal{E}, i, f}^{n+1}\right) \mu_{f}^{k}+\delta_{t} \sum_{i=1}^{L} Q_{i, k}^{n+1}
$$

where the fluxes of potential energy $\mathcal{G}_{\mathcal{E}, i, f}^{n}$ and $\mathcal{H}_{\mathcal{E}, i, f}^{n}$ are defined by (2.5) and the work of the force $Q_{i, k}^{n}$ is given by (2.7). The numerical source term $R_{\mathcal{E}, i, f}^{n+1}$ is defined by

$$
R_{\mathcal{E}, i, f}^{n+1}:=\left[\phi_{i}^{n+1}\right]_{k}^{k_{f}}\left(\mathcal{F}_{i, f}^{n+1}-\left(\rho_{i}^{n+1} U_{i}^{n}\right)_{f}\right) \cdot N_{k}^{k_{f}}
$$

Proof. Let us consider the potential energy at the intermediate time step $\mathcal{E}_{k}^{n+s}=$ $\mathcal{E}\left(s \boldsymbol{\rho}_{k}^{n+1}+(1-s) \boldsymbol{\rho}_{k}^{n}, X_{k}\right)$. Accordingly to the mean value theorem, there exists $0<$ $s_{k}^{\star}<1$ such that

$$
\begin{aligned}
\mathcal{E}_{k}^{n+1} & =\mathcal{E}_{k}^{n}+\partial_{s} \mathcal{E}_{k}^{n+1}-\frac{1}{2} \partial_{s s}^{2} \mathcal{E}_{k}^{n+s_{k}^{\star}} \\
& =\mathcal{E}_{k}^{n}+\sum_{i=1}^{L}\left(\rho_{i, k}^{n+1}-\rho_{i, k}^{n}\right) \phi_{i, k}^{n+1}-\frac{1}{2} \sum_{i=1}^{L} \sum_{j=1}^{L}\left(\rho_{i, k}^{n+1}-\rho_{i, k}^{n}\right) \mathbf{H}_{i j, k}^{n+s_{k}^{\star}}\left(\rho_{j, k}^{n+1}-\rho_{j, k}^{n}\right)
\end{aligned}
$$

with $\mathbf{H}_{i j, k}^{n+s_{k}^{\star}}=\mathbf{H}_{i j}\left(s_{k}^{\star} \boldsymbol{\rho}_{k}^{n+1}+\left(1-s_{k}^{\star}\right) \boldsymbol{\rho}_{k}^{n}, X_{k}\right)$. Under the assumption of (1.1), the last term is nonnegative. Then, using the mass scheme $\left(S_{\varepsilon}^{\delta_{t}} \cdot a\right)$, the following bound holds

$$
\mathcal{E}_{k}^{n+1} \leq \mathcal{E}_{k}^{n}-\frac{\delta_{t}}{\ell_{k}} \sum_{f \in \mathbb{F}_{k}} \sum_{i=1}^{L} \phi_{i, k}^{n+1} \mathcal{F}_{i, f}^{n+1} \cdot N_{k}^{k_{f}} \mu_{f}^{k}
$$

Finally, the exchange of energy $Q_{i, k}^{n+1}$ is added to the right-hand-side and the rest is split into an anti-symmetric flux $\mathcal{G}_{\mathcal{E}, i, f}^{n+1}$ and a symmetric residual $R_{\mathcal{E}, i, f}^{n+1}$ to get the announced result.

Proof. [of Theorem 2.3] The definition of the numerical mass flux $\left(S_{\varepsilon}^{\delta_{t}} . c\right)$ is motivated by the leading form of the potential energy residual (2.10). More precisely, injecting $\left(S_{\varepsilon}^{\delta_{t}} . c\right)$ in (2.10) and summing with (2.7), the mechanic source term reads

$$
R_{\mathcal{K}, i, f}^{n+1}+\frac{R_{\mathcal{E}, i, f}^{n+1}}{\varepsilon^{2}}=(1-\gamma) \delta_{t}\left(\frac{\rho_{i}^{n+1}}{\ell}\right)_{f}\left\|\frac{\left[\phi_{i}^{n+1}\right]_{k}^{k_{f}}}{\varepsilon^{2}}\right\|^{2} .
$$


We conclude the dissipation law of the mechanical energy (2.3).

Note that the bound (2.3) is a dissipation law since the fluxes $\mathcal{G}_{\mathcal{K}, i, f}^{n+1} \cdot N_{k}^{k_{f}}, \mathcal{G}_{\mathcal{E}, i, f}^{n+1}$. $N_{k}^{k_{f}}, \mathcal{H}_{\mathcal{K}, i, f}^{n+1}$ and $\mathcal{H}_{\mathcal{E}, i, f}^{n+1}$ are anti-symmetric with respect to the control volume. More precisely, summing inequality (2.3) over the cells $k \in \mathbb{T}$ leads to the energy decay law, which can be considered as a nonlinear stability argument. The mechanical energy dissipation leads to the following bound of the variation of potential, i.e.

$$
(\gamma-1) \sum_{n=1}^{T} \delta_{t} \sum_{f \in \mathbb{F}}\left(\frac{\rho_{i}^{n}}{\ell}\right)_{f}\left\|\frac{\left[\phi_{i}^{n}\right]_{k}^{k_{f}}}{\varepsilon^{2}}\right\|^{2} \leq \sum_{k \in \mathbb{T}} \ell_{k} E_{k}^{0} .
$$

2.3. Conservation law and steady states at rest. The following section is devoted to the translation of the physical properties Proposition 1.1 and Proposition 1.2 of the model $\left(M_{\varepsilon}^{t}\right)$ at the discrete level.

Proposition 2.6 (Discrete conservation of the mass). The discrete mass is conserved by the CPR scheme $\left(S_{\varepsilon}^{\delta_{t}}\right)$. More precisely

$$
\sum_{k \in \mathbb{T}}|k| \rho_{i, k}^{n}=\sum_{k \in \mathbb{T}}|k| \rho_{i, k}^{0} .
$$

The total momentum conservation Proposition (1.2) is not easy to satisfy at the discrete level for any potential mapping. The following result Proposition (2.7) shows the conservation in the case of a quadratic energy and as a consequence with constant Hessian. However, many physical models have a quadratic energy. For example, the multilayer shallow water model satisfies this assumption and more precisely the Hessian is given by $\mathbf{H}_{i j}=\frac{g}{\varrho_{\max (i, j)}}$.

Proposition 2.7 (Discrete conservation of the total momentum). Assume that the Hessian is a constant and symmetric matrix, i.e.

$$
\partial_{\rho_{i}} \mathbf{H}=0, \quad \nabla \mathbf{H}=0 \quad \text { and } \quad \mathbf{H}_{i j}=\mathbf{H}_{j i} .
$$

Then the discrete total momentum satisfies the following balance

$$
\begin{aligned}
\sum_{i=1}^{L} \rho_{i, k}^{n+1} U_{i, k}^{n+1}= & \sum_{i=1}^{L} \rho_{i, k}^{n} U_{i, k}^{n}-\frac{\delta_{t}}{\ell_{k}} \sum_{f \in \mathbb{F}_{k}} \sum_{i=1}^{L}\left(\left(\rho_{i}^{n+1}\right)_{f}\left(\phi_{i}^{n+1}\right)_{f}-\frac{1}{2}\left(\rho_{i}^{n+1} \phi_{i}^{n+1}\right)_{f}\right) \mu_{f}^{k} \\
& -\frac{\delta_{t}}{\ell_{k}} \sum_{f \in \mathbb{F}_{k}} \sum_{i=1}^{L}\left(U_{i, k}^{n}\left(\mathcal{F}_{i, f}^{n+1} \cdot N_{f}^{k}\right)^{+}-U_{i, k_{f}}^{n}\left(\mathcal{F}_{i, f}^{n+1} \cdot N_{f}^{k}\right)^{-}\right) \mu_{f}^{k} .
\end{aligned}
$$

It follows that the discrete global momentum is conserved by the CPR scheme $\left(S_{\varepsilon}^{\delta_{t}}\right)$. More precisely

$$
\sum_{k \in \mathbb{T}} \sum_{i=1}^{L}|k| \rho_{i, k}^{n} U_{i, k}^{n}=\sum_{k \in \mathbb{T}} \sum_{i=1}^{L}|k| \rho_{i, k}^{0} U_{i, k}^{0} .
$$

Proof. Summing the momentum scheme $\left(S_{\varepsilon}^{\delta_{t}} . b\right)$ over the mass $1 \leq i \leq L$, the nonconservative term becomes

$$
\sum_{i=1}^{L} \rho_{i, k}^{n+1} \sum_{f \in \mathbb{F}_{k}} \frac{\left(\phi_{i}^{n+1}\right)_{f}}{\varepsilon^{2}} N_{f}^{k} \mu_{f}^{k}=\sum_{f \in \mathbb{F}_{k}} \sum_{i=1}^{L} \frac{\rho_{i, k}^{n+1} \phi_{i, k_{f}}^{n+1}}{2 \varepsilon^{2}} N_{f}^{k} \mu_{f}^{k}
$$


Since the Hessian is constant, the potential can be written under the form $\phi_{i, k_{f}}^{n+1}=$ $\sum_{j=1}^{L} \mathbf{H}_{i j} \rho_{j, k_{f}}^{n+1}$ and since the Hessian is symmetric, i.e. $\mathbf{H}_{i j}=\mathbf{H}_{j i}$, it reads

$$
\sum_{i=1}^{L} \rho_{i, k}^{n+1} \phi_{j, k_{f}}^{n+1}=\sum_{i=1}^{L} \sum_{j=1}^{L} \rho_{i, k}^{n+1} \mathbf{H}_{i j} \rho_{j, k_{f}}^{n+1}=\sum_{i=1}^{L} \sum_{j=1}^{L} \rho_{j, k_{f}}^{n+1} \mathbf{H}_{j i} \rho_{i, k}^{n+1}=\sum_{j=1}^{L} \rho_{j, k_{f}}^{n+1} \phi_{j, k}^{n+1} .
$$

Then the half sum of the left-hand side and the right-hand side leads to

$$
\sum_{i=1}^{L} \rho_{i, k}^{n+1} \sum_{f \in \mathbb{F}_{k}} \frac{\left(\phi_{i}^{n+1}\right)_{f}}{\varepsilon^{2}} N_{f}^{k} \mu_{f}^{k}=\sum_{f \in \mathbb{F}_{k}} \sum_{i=1}^{L} \frac{\rho_{i, k}^{n+1} \phi_{i, k_{f}}^{n+1}+\rho_{i, k_{f}}^{n+1} \phi_{i, k}^{n+1}}{4 \varepsilon^{2}} N_{f}^{k} \mu_{f}^{k} .
$$

Finally using the equality $\frac{\rho_{i, k}^{n+1} \phi_{i, k_{f}}^{n+1}+\rho_{i, k_{f}}^{n+1} \phi_{i, k}^{n+1}}{4}=\left(\rho_{i}^{n+1}\right)_{f}\left(\phi_{i}^{n+1}\right)_{f}-\frac{1}{2}\left(\rho_{i}^{n+1} \phi_{i}^{n+1}\right)_{f}$, we get the announced result.

Proposition 2.8 (Well-balanced). The CPR scheme $\left(S_{\varepsilon}^{\delta_{t}}\right)$ preserves the discrete steady states at rest defined by $U_{i, k}^{n}=0$ and $\phi_{i, k}^{n}=\bar{\phi}_{i}$.

Proof. Assume that the discrete unknowns at the $n^{\text {th }}$ time iteration satisfy the the steady state at rest. Let us consider first the mass scheme $\left(S_{\varepsilon}^{\delta_{t}} \cdot a\right)$. Since the discharge and the variation of potential vanish $\left(\rho_{i}^{n+1} U_{i}^{n}\right)_{f}=0$ and $\left[\phi_{i}^{n}\right]_{k}^{k_{f}}=0$, the scheme can be written as

$$
\rho_{i, k}^{n+1}-\gamma \frac{\delta_{t}^{2}}{\ell_{k}} \sum_{f \in \mathbb{F}_{k}}\left(\frac{\rho_{i}^{n+1}}{\ell}\right)_{f} \frac{\left[\phi_{i}^{n+1}\right]_{k}^{k_{f}}}{\varepsilon^{2}} \mu_{f}^{k}=\rho_{i, k}^{n}-\gamma \frac{\delta_{t}^{2}}{\ell_{k}} \sum_{f \in \mathbb{F}_{k}}\left(\frac{\rho_{i}^{n}}{\ell}\right)_{f} \frac{\left[\phi_{i}^{n}\right]_{k}^{k_{f}}}{\varepsilon^{2}} \mu_{f}^{k} .
$$

The unique solution is $\rho_{i, k}^{n+1}=\rho_{i, k}^{n}$, which implies $\phi_{i, k}^{n+1}=\bar{\phi}_{i}$. Finally, the velocity at time iteration $n+1$ vanishes $U_{i, k}^{n+1}=0$ since the numerical flux $\mathcal{F}_{i, f}^{n+1}$ and the potential variation $\left[\phi_{i}^{n+1}\right]_{k}^{k_{f}}$ vanish. $\square$

Let us highlight that the CPR scheme $\left(S_{\varepsilon}^{\delta_{t}}\right)$ is stable and consistent without condition on the velocity difference. Indeed, for several physical model under the form $\left(M_{\varepsilon}^{t}\right)$, an additional condition on the velocity difference is required to proof the hyperbolicity of the system, see $[13,30,31]$ in the framework of free surface flows. Obviously if this condition is not satisfied, the solution of the CPR scheme $\left(S_{\varepsilon}^{\delta_{t}}\right)$ is well-defined but is not relevant as a mathematical solution of the PDE system.

3. Asymptotic regimes. In the following section, the behavior of the scheme in the regime $\varepsilon \ll 1$ is analyzed. Fig. 1 illustrate the following properties. The advection model $\left(M_{\varepsilon}^{t}\right)$ has two asymptotic behaviors relevant depending on the time scale considered.

3.1. Fine time scale: the acoustic regime $\left(M_{0}^{\tau}\right)$. In the following section, the behavior of the solution of $\left(M_{\varepsilon}^{t}\right)$ for a small time scale $t=\varepsilon \tau$ and in the limit where $\varepsilon$ goes to zero is analyzed. We refer to [43] for the derivation and the analytical results about the acoustic regime.

For any fixed $\bar{\phi} \in \mathbb{R}^{L}$, the main masses $\overline{\boldsymbol{\rho}}_{k}$ are defined such that $\phi_{i}\left(\overline{\boldsymbol{\rho}}_{k}, X_{k}\right)=\bar{\phi}_{i}$ 
and we consider the following scheme

$\left(S_{0}^{\delta_{\tau}}\right)$

$$
\begin{aligned}
& \widetilde{\phi}_{i, k}^{n+1}=\widetilde{\phi}_{i, k}^{n}-\frac{\delta_{\tau}}{\ell_{k}} \sum_{j=1}^{L}\left(\overline{\mathbf{H}}_{i j}\right)_{k} \sum_{f \in \mathbb{F}_{k}}\left(\left(\bar{\rho}_{j} \widetilde{U}_{j}^{n}\right)_{f} \cdot N_{k}^{k_{f}}-\gamma \delta_{\tau}\left(\frac{\bar{\rho}_{j}}{\ell}\right)_{f}\left[\widetilde{\phi}_{j}^{n+1}\right]_{k}^{k_{f}}\right) \mu_{f}^{k} \\
& \widetilde{U}_{i, k}^{n+1}=\widetilde{U}_{i, k}^{n}-\frac{\delta_{\tau}}{\ell_{k}} \sum_{f \in \mathbb{F}_{k}}\left(\widetilde{\phi}_{i}^{n+1}\right)_{f} N_{k}^{k_{f}} \mu_{f}^{k}
\end{aligned}
$$

with $\left(\overline{\mathbf{H}}_{i j}\right)_{k}:=\mathbf{H}_{i j}\left(\overline{\boldsymbol{\rho}}_{k}, X_{k}\right)$. The following consistency result holds

Proposition 3.1. Assume that the solution is smooth enough and the tessellation is a regular cartesian grid with a space step $\delta_{x}$. Then the numerical strategy $\left(S_{0}^{\delta_{\tau}}\right)$ is consistent with the acoustic regime $\left(M_{0}^{\tau}\right)$. More precisely the modified system reads

$$
\left\{\begin{aligned}
\partial_{\tau} \widetilde{\phi}_{i}+\sum_{j=1}^{L} \overline{\mathbf{H}}_{i j} \nabla \cdot\left(\bar{\rho}_{j} \widetilde{U}_{j}\right) & =\frac{\delta_{\tau}}{2} \sum_{j=1}^{L} \overline{\mathbf{H}}_{i j} \nabla \cdot\left((2 d \gamma-1) \bar{\rho}_{j} \nabla \widetilde{\phi}_{j}\right)+O\left(\delta_{\tau}^{2}, \delta_{x}^{2}\right) \\
\partial_{\tau} \widetilde{U}_{i}+\nabla \widetilde{\phi}_{i} & =-\frac{\delta_{\tau}}{2} \sum_{j=1}^{L} \nabla\left(\overline{\mathbf{H}}_{i j} \nabla \cdot\left(\bar{\rho}_{j} \widetilde{U}_{j}\right)\right)+O\left(\delta_{\tau}^{2}, \delta_{x}^{2}\right) .
\end{aligned}\right.
$$

Proof. The proof is similar to the proof of Proposition 2.2.

Proposition 3.2. Assume that the Hessian of the potential energy is wellconditioned independently of $\varepsilon$, i.e. $\kappa=\underset{\varepsilon \rightarrow 0}{O}(1)$, and the discretized initial condition satisfies

$$
\rho_{i, k}^{0}=\underset{\varepsilon \rightarrow 0}{O}(1), \quad U_{i, k}^{0}=\underset{\varepsilon \rightarrow 0}{O}(\varepsilon) \quad \text { and } \quad \phi_{i}\left(\boldsymbol{\rho}_{k}^{0}, X_{k}\right)=\bar{\phi}_{i}+\underset{\varepsilon \rightarrow 0}{O}(\varepsilon) .
$$

with the parameter $\bar{\rho}_{i, k}$ such that the potential $\bar{\phi}_{i}=\phi_{i}\left(\overline{\boldsymbol{\rho}}_{k}, X_{k}\right)$ is space independent. Then the CPR scheme $\left(S_{\varepsilon}^{\delta_{t}}\right)$ with the time step scaling $\delta_{t}=\underset{\varepsilon \rightarrow 0}{O}(\varepsilon)$ tends to the scheme $\left(S_{0}^{\delta_{\tau}}\right)$ in the limit where $\varepsilon$ goes to zero. More precisely, for any time iteration $n \in \mathbb{N}$, for any control volume $k \in \mathbb{T}$ scheme differences read

$$
\rho_{i, k}^{n}-\left(\bar{\rho}_{i, k}+\varepsilon \widetilde{\rho}_{i, k}^{n}\right)=\underset{\varepsilon \rightarrow 0}{O}\left(\varepsilon^{2}\right) \quad \text { and } \quad U_{i, k}^{n}-\varepsilon \widetilde{U}_{i, k}^{n}=\underset{\varepsilon \rightarrow 0}{O}\left(\varepsilon^{2}\right)
$$

with $\left(\rho_{i, k}^{n}, U_{i, k}^{n}\right)$, the solution of the CPR scheme $\left(S_{\varepsilon}^{\delta_{t}}\right)$ and $\widetilde{\boldsymbol{\rho}}_{k}^{n}=\overline{\mathbf{H}}_{k}^{-1} \widetilde{\boldsymbol{\phi}}_{k}^{n}$ with $\left(\widetilde{\phi}_{i, k}^{n}, \widetilde{U}_{i, k}^{n}\right)$, the solution of the scheme $\left(S_{0}^{\delta_{\tau}}\right)$ with the initial condition $\widetilde{\rho}_{i, k}^{0}=\rho_{i, k}^{0}$, $\widetilde{U}_{i, k}^{0}=U_{i, k}^{0}$ and $\overline{\mathbf{H}}_{k}^{-1}$ is the inverse matrix of $\left(\overline{\mathbf{H}}_{k}\right)_{i, j}=\partial_{\rho_{j}} \phi_{i}\left(\overline{\boldsymbol{\rho}}_{k}, X_{k}\right)$.

Proof. First the time of the numerical scheme is rescaled. Note that since $U_{i, k}^{n}$ is a velocity, the rescaled velocity becomes $u_{i, k}^{n}:=\frac{U_{i, k}^{n}}{\varepsilon}$. Assume the following scaling of the numerical unknowns at the previous iteration $n$

$$
\phi_{i, k}^{n}=\phi_{i}\left(\boldsymbol{\rho}_{k}^{n}, X_{k}\right)=\bar{\phi}_{i}+\underset{\varepsilon \rightarrow 0}{O}(\varepsilon) \quad \text { and } \quad u_{i, k}^{n}=\underset{\varepsilon \rightarrow 0}{O}(1)
$$

which is true by hypothesis for the initial condition. The main term of the mass scheme $\left(S_{\varepsilon}^{\delta_{t}} . a\right)$ (with $\left.\delta_{\tau}:=\frac{\delta_{t}}{\varepsilon}\right)$ can be written as

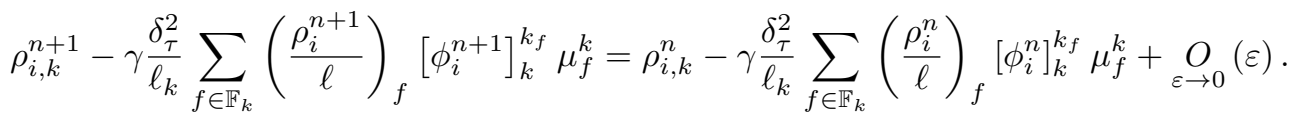


Since the Hessian is well-conditioned, the potential reads

$$
\phi_{i}\left(\boldsymbol{\rho}_{k}^{n}+\underset{\varepsilon \rightarrow 0}{O}(\varepsilon), X_{k}\right)=\phi_{i}\left(\boldsymbol{\rho}_{k}^{n}, X_{k}\right)+\sum_{j=1}^{L}\left(\mathbf{H}_{i j}\right)_{k}^{n} \underset{\varepsilon \rightarrow 0}{O}(\varepsilon)=\bar{\phi}_{i}^{n}+\underset{\varepsilon \rightarrow 0}{O}(\varepsilon)
$$

with $\left(\mathbf{H}_{i j}\right)_{k}^{n}=\mathbf{H}_{i j}\left(\boldsymbol{\rho}_{k}^{n}, X_{k}\right)$. Then the solution for the main term is $\rho_{i, k}^{n+1}=\rho_{i, k}^{n}+$ $\underset{\varepsilon \rightarrow 0}{O}(\varepsilon)$. We conclude by induction that the main term of the mass is constant in time, then the main term of the potential is constant in time and in space. Then the numerical unknowns of the CPR scheme $\left(S_{\varepsilon}^{\delta_{t}}\right)$ are expanded as $\rho_{i, k}^{n}=\bar{\rho}_{i, k}+\varepsilon r_{i, k}^{n}$. The potential can be expanded as

$$
\phi_{i}\left(\overline{\boldsymbol{\rho}}_{k}+\varepsilon \mathbf{r}_{k}^{n}, X_{k}\right)=\bar{\phi}_{i}+\varepsilon p_{i, k}^{n}+\underset{\varepsilon \rightarrow 0}{O}\left(\varepsilon^{2}\right)
$$

with $p_{i, k}^{n}=\sum_{j=1}^{L}\left(\overline{\mathbf{H}}_{i j}\right)_{k} r_{j, k}^{n}$ and the main part of the Hessian $\left(\overline{\mathbf{H}}_{i j}\right)_{k}=\mathbf{H}_{i j}\left(\overline{\boldsymbol{\rho}}_{k}, X_{k}\right)=$ $\partial_{\rho_{j}} \phi_{i}\left(\overline{\boldsymbol{\rho}}_{k}, X_{k}\right)$ is time independent. Composing $\left(S_{\varepsilon}^{\delta_{t}} . a\right)$ by the Hessian $\left(\overline{\mathbf{H}}_{i j}\right)_{k}$ and summing over the components, the perturbation of the potential reads

$$
\frac{p_{i, k}^{n+1}-p_{i, k}^{n}}{\delta_{\tau}}+\sum_{j=1}^{L} \frac{\left(\overline{\mathbf{H}}_{i j}\right)_{k}}{\ell_{k}} \sum_{f \in \mathbb{F}_{k}}\left(\left(\bar{\rho}_{j} u_{j}^{n}\right)_{f} \cdot N_{k}^{k_{f}}-\gamma \delta_{\tau}\left(\frac{\bar{\rho}_{j}}{\ell}\right)_{f}\left[p_{j, f}^{n+1}\right]_{k}^{k_{f}}\right) \mu_{f}^{k}=\underset{\varepsilon \rightarrow 0}{O}(\varepsilon) .
$$

Similarly, the main term of the momentum scheme $\left(S_{\varepsilon}^{\delta_{t}} . b\right)$ reads

$$
u_{i, k}^{n+1}=u_{i, k}^{n}-\frac{\delta_{\tau}}{\ell_{k}} \sum_{f \in \mathbb{F}_{k}}\left(p_{i}^{n+1}\right)_{f} N_{k}^{k_{f}} \mu_{f}^{k}+\underset{\varepsilon \rightarrow 0}{O}(\varepsilon) .
$$

We conclude that $\left(\widetilde{\phi}_{i, k}^{n}, \widetilde{U}_{i, k}^{n}\right)$ is an approximation in order of $\varepsilon$ of $\left(p_{i, k}^{n}, u_{i, k}^{n}\right)$.

3.2. Large time scale: the incompressible regime $\left(M_{0}^{t}\right)$. In the following section, the behavior of the solution of $\left(M_{\varepsilon}^{t}\right)$ for a large enough time scale, i.e. $t=$ $\underset{\varepsilon \rightarrow 0}{O}(1)$ and when $\varepsilon$ goes to zero is investigated. We refer to [23, 24, 28] for the derivation and the analytical results in the incompressible regime. This regime is relevant for many applications such are nuclear reactor [5] and oceanography [40].

For any fixed $\bar{\phi} \in \mathbb{R}^{L}$, the main term of the masses $\overline{\boldsymbol{\rho}}_{k}$ is defined such that $\phi_{i}\left(\overline{\boldsymbol{\rho}}_{k}, X_{k}\right)=\bar{\phi}_{i}$. Let us now consider the following scheme

$\left(S_{0}^{\delta_{t}} \cdot a\right)$

$$
\begin{aligned}
\bar{U}_{i, k}^{n+1}= & \bar{U}_{i, k}^{n}-\frac{\delta_{t}}{\ell_{k}} \sum_{f \in \mathbb{F}_{k}}\left[\psi_{i}^{n+1}\right]_{k}^{k_{f}} N_{k}^{k_{f}} \mu_{f}^{k} \\
& -\frac{\delta_{t}}{\bar{\rho}_{i, k} \ell_{k}} \sum_{f \in \mathbb{F}_{k}}\left(\bar{U}_{i, k}^{n}\left(\overline{\mathcal{F}}_{i, f}^{n+1} \cdot N_{k}^{k_{f}}\right)^{+}-\bar{U}_{i, k_{f}}^{n}\left(\overline{\mathcal{F}}_{i, f}^{n+1} \cdot N_{k}^{k_{f}}\right)^{-}\right) \mu_{f}^{k} .
\end{aligned}
$$

The variation of the perturbation of the potential $\left[\psi_{i}^{n+1}\right]_{k}^{k_{f}}$ is computed by

$$
\sum_{f \in \mathbb{F}_{k}} \overline{\mathcal{F}}_{i, f}^{n+1} \cdot N_{k}^{k_{f}} \mu_{f}^{k}=0
$$

with the asymptotic mass flux $\overline{\mathcal{F}}_{i, f}^{n+1}:=\left(\bar{\rho}_{i} \bar{U}_{i}^{n}\right)_{f}-\gamma \delta_{t}\left(\frac{\bar{\rho}_{i}}{\ell}\right)_{f}\left[\psi_{i}^{n+1}\right]_{k}^{k_{f}}$. The numerical scheme $\left(S_{0}^{\delta_{t}} \cdot b\right)$ is a discretization of the constraint $\nabla \cdot\left(\bar{\rho}_{i} \bar{U}_{i}\right)=0$. Thanks to the 
regularization, the numerical scheme $\left(S_{0}^{\delta_{t}}\right)$ can be classified as a pseudo-compressible Petrov-Galerkin method, see [39].

Proposition 3.3. Assume that the solution is smooth enough and the tessellation is a regular cartesian grid with a space step $\delta_{x}$. Then the numerical strategy $\left(S_{0}^{\delta_{t}}\right)$ is consistent with the incompressible regime $\left(M_{0}^{t}\right)$. More precisely the modified system reads

$$
\left\{\begin{array}{l}
\nabla \cdot\left(\bar{\rho}_{i} \bar{U}_{i}\right)=\frac{\delta_{t}}{2} \nabla \cdot\left((2 d \gamma-1) \bar{\rho}_{i} \nabla \psi_{i}-\bar{\rho}_{i}\left(\bar{U}_{i} \cdot \nabla\right) \bar{U}_{i}\right)+O\left(\delta_{t}^{2}, \delta_{x}^{2}\right) \\
\partial_{t} \bar{U}_{i}+\left(\bar{U}_{i} \cdot \nabla\right) \bar{U}_{i}=-\nabla \psi_{i}-\frac{\delta_{t}}{2} \partial_{t}\left(\nabla \psi_{i}\right)+\delta_{t} d \gamma\left(\nabla \psi_{i} \cdot \nabla\right) \bar{U}_{i}+O\left(\delta_{t}^{2}, \delta_{x}\right)
\end{array}\right.
$$

Proof. The proof is similar to the proof of Proposition 2.2.

Proposition 3.4. Assume that the Hessian of the potential energy is wellconditioned independently of $\varepsilon$, i.e. $\kappa=\underset{\varepsilon \rightarrow 0}{O}(1)$, and the discretized initial condition satisfies

$$
\rho_{i, k}^{0}=\underset{\varepsilon \rightarrow 0}{O}(1), \quad U_{i, k}^{0}=\underset{\varepsilon \rightarrow 0}{O}(1) \quad \text { and } \quad \phi_{i}\left(\boldsymbol{\rho}_{k}^{0}, X_{k}\right)=\bar{\phi}_{i}+\underset{\varepsilon \rightarrow 0}{O}\left(\varepsilon^{2}\right) .
$$

Then the CPR scheme $\left(S_{\varepsilon}^{\delta_{t}}\right)$ with the time step scaling $\delta_{t}=\underset{\varepsilon \rightarrow 0}{O}(1)$ tends to the scheme $\left(S_{0}^{\delta_{t}}\right)$ and when $\varepsilon$ goes to zero. More precisely for any time iteration $n \in \mathbb{N}$ and any control volume $k \in \mathbb{T}$, the scheme differences read

$$
\rho_{i, k}^{n}-\bar{\rho}_{i, k}=\underset{\varepsilon \rightarrow 0}{O}\left(\varepsilon^{2}\right) \quad \text { and } \quad U_{i, k}^{n}-\bar{U}_{i, k}^{n}=\underset{\varepsilon \rightarrow 0}{O}\left(\varepsilon^{2}\right)
$$

with $\left(\rho_{i, k}^{n}, U_{i, k}^{n}\right)$ the solution of the CPR scheme $\left(S_{\varepsilon}^{\delta_{t}}\right)$, the parameter $\bar{\rho}_{i, k}$ satisfies $\phi_{i}\left(\overline{\boldsymbol{\rho}}_{k}, X_{k}\right)=\phi_{i}^{0}$ and $\bar{U}_{i, k}^{n}$ the solution of the scheme $\left(S_{0}^{\delta_{t}}\right)$ with the initial condition $\bar{U}_{i, k}^{0}=U_{i, k}^{0}$.

Proof. Since the masses are positive (Proposition 2.1) and the numerical scheme conserves the mass (Proposition 2.6), the mass in a control volume $\rho_{i, k}^{n+1}$ is bounded by the initial condition, i.e. $\ell_{k} \rho_{i, k}^{n+1} \leq \sum_{j \in \mathbb{T}} \ell_{j} \rho_{i, j}^{0}=\underset{\varepsilon \rightarrow 0}{O}(1)$. Then the main term of the mass scheme $\left(S_{\varepsilon}^{\delta_{t}} . a\right)$ reads

$$
-\gamma \frac{\delta_{t}^{2}}{\ell_{k}} \sum_{f \in \mathbb{F}_{k}}\left(\frac{\rho_{i}^{n+1}}{\ell}\right)_{f} \frac{\left[\phi_{i}^{n+1}\right]_{k}^{k_{f}}}{\varepsilon^{2}} \mu_{f}^{k}=\underset{\varepsilon \rightarrow 0}{O}(1) .
$$

We conclude that for any $f \in \mathbb{F}$, the variation of the potential reads $\left[\phi_{i}^{n+1}\right]_{k}^{k_{f}}=$ $\underset{\varepsilon \rightarrow 0}{O}\left(\varepsilon^{2}\right)$. It follows that the main term of the potential is constant in space, i.e. $\phi_{i, k}^{n}=\phi_{i}^{n}+\underset{\varepsilon \rightarrow 0}{O}\left(\varepsilon^{2}\right)$. On the another hand, for any $X \in \Omega$, since the Hessian $\mathbf{H}$ is positive-definite, the application $\boldsymbol{\phi}(\boldsymbol{\rho}, X)$ is a diffeomorphism according to the inverse function theorem. The mass at the intermediate time is defined by $\rho_{i, k}^{n+s}=$ $\rho_{i}\left(s \boldsymbol{\phi}_{k}^{n+1}+(1-s) \boldsymbol{\phi}_{k}^{n}, X_{k}\right)$ with $\boldsymbol{\rho}(\boldsymbol{\phi}, X)$ the inverse function for a given $X$ of $\boldsymbol{\phi}(\boldsymbol{\rho}, X)$. According to the mean value theorem, there exists an intermediate time $0<s_{i, k}^{\star}<1$ such that

$$
\rho_{i, k}^{n+1}-\rho_{i, k}^{n}=\sum_{j=1}^{L}\left(\mathbf{H}_{i j}^{-1}\right)_{k}^{n+s_{i, k}^{\star}}\left(\phi_{j, k}^{n+1}-\phi_{j, k}^{n}\right)
$$


with $\left(\mathbf{H}_{i j}^{-1}\right)_{k}^{n+s_{i, k}^{\star}}=\mathbf{H}_{i j}^{-1}\left(s_{i, k}^{\star} \boldsymbol{\rho}_{k}^{n+1}+\left(1-s_{i, k}^{\star}\right) \boldsymbol{\rho}_{k}^{n}, X_{k}\right)$ positive-definite. After summing over the control volume, since the main term of the potential is constant in space and using the mass conservation, the time variation of the potential reads

$$
\sum_{j=1}^{L} \mathbf{M}_{i j}\left(\phi_{j}^{n+1}-\phi_{j}^{n}\right)=\underset{\varepsilon \rightarrow 0}{O}\left(\varepsilon^{2}\right) .
$$

with $\mathbf{M}_{i j}=\left(\sum_{k \in \mathbb{T}} \ell_{k}\left(\mathbf{H}_{i j}^{-1}\right)_{k}^{n+s_{i, k}^{\star}}\right)$ positive-definite as a finite sum of positivedefinite matrices. Likewise, $\mathbf{M}$ is well-conditioned independently of $\varepsilon$, i.e. $\kappa_{\mathbf{M}}=$ $\underset{\varepsilon \rightarrow 0}{O}(\kappa)=\underset{\varepsilon \rightarrow 0}{O}(1)$ with $\kappa_{\mathbf{M}}$ the condition number of $\mathbf{M}$. We conclude that the main term of the potential is constant in space and in time, i.e. $\phi_{i, k}^{n+1}=\bar{\phi}_{i}+\underset{\varepsilon \rightarrow 0}{O}\left(\varepsilon^{2}\right)$. It follows that the mass is close enough to the parameter of the asymptotic scheme $\bar{\rho}_{i, k}$, i.e. $\rho_{i, k}^{n}=\bar{\rho}_{i, k}+\underset{\varepsilon \rightarrow 0}{O}\left(\varepsilon^{2}\right)$. Accordingly to the mean value theorem, there exists an intermediate time $0<s_{i, k}<1$ such that

$$
\phi_{i, k}^{n+1}-\phi_{i, k}^{n}=\sum_{j=1}^{L}\left(\mathbf{H}_{i j}\right)_{k}^{n+s_{i, k}}\left(\rho_{j, k}^{n+1}-\rho_{j, k}^{n}\right)
$$

with $\left(\mathbf{H}_{i j}\right)_{k}^{n+s_{i, k}}=\mathbf{H}_{i j}\left(s_{i, k} \boldsymbol{\rho}_{k}^{n+1}+\left(1-s_{i, k}\right) \boldsymbol{\rho}_{k}^{n}, X_{k}\right)$. Using the mass conservation $\left(S_{\varepsilon}^{\delta_{t}} \cdot a\right)$, it yields the divergence free condition $\left(S_{0}^{\delta_{t}} \cdot b\right)$ with a second order perturbation. In addition, replacing the mass $\rho_{i, k}^{n+1}$ by the parameter $\bar{\rho}_{i, k}$ in the momentum scheme $\left(S_{\varepsilon}^{\delta_{t}} . b\right)$, it leads to the asymptotical scheme of the velocity $\left(S_{0}^{\delta_{t}}\right)$ with a second order perturbation.

4. Numerical results. The current section is devoted to the illustration of the analytical results obtained in Section 2 and 3. The following simulations are presented in 1 space dimension, in the framework of free surface flows, see Section 1.

In term of runtime, the CPR scheme is not efficient in the case of large dimensionless parameter $\varepsilon \gg 1$. In this case, the Gogunov's schemes are very efficient. However, the CPR scheme is very efficient in the case of small dimensionless parameter $\varepsilon \ll 1$, unlike the Gogunov's scheme. In the case of dimensionless parameter $\varepsilon$ in order of 1 , the runtime of the schemes are comparable. Note that according to the CFL condition (2.2), the CPR scheme at the steady state at rest is stable for any time step $\delta_{t}>0$. Numerical convergence tests were performed and the consistency errors correspond to Property 2.2 (first order), Property 3.1 (second order) and Property 3.3 (first order), depending on the regime.

Several simulations of the steady state at rest, the so-called lake at rest in the framework of free surface flow, are performed with various bottom elevations, continuous or not, several layers and small or large density variations to validate Property 2.8 . The proposition is satisfied in any situations where the bottom elevation is completely immersed, i.e. $h_{L, k}^{0}>0$ and the density is well-stratified, with the error of the resolution method of the linear system. In practice, the deviation of the layer thickness is of the order of $10^{-15}$ and the maximum of the velocities is of the order of $10^{-13}$.

4.1. Simulation of oscillating surfaces. This test case is the simulation of oscillating layers around the steady state at rest in an infinite domain. More precisely the initial condition is given by

$$
h_{1}^{0}=10^{3}+\cos (2 \pi x), \quad u_{1}^{0}=0, \quad \forall 2 \leq i \leq L=5, \quad h_{i}^{0}=10^{3}, \quad u_{i}^{0}=0
$$


and $B=0$. The density of the layer is given by $\varrho_{i}=10+i$. These parameters leads to a Froude number $\varepsilon=10^{-3}$ witch is of the same order as in the ocean context. The simulation can be seen as a simplified modeling of ocean flow, neglecting several external forces such as the Coriolis force and the atmospheric pressure variation. The objective of the current test case is to compare the CPR scheme in the low-Froude number regime to an analytical solution of the asymptotic model $\left(M_{0}^{\tau}\right)$, obtained by D'Alembert's formulae. The eigenvalues and the eigenvectors required for D'Alembert's formulae are determined numerically in the following using a QR decomposition with Givens rotations. The solution of the asymptotic model is a superposition of five waves with the speeds $\left(c_{1}, c_{2}, c_{3}, c_{4}\right) \approx(14,17,23,43,215)$. Several of them can clearly be identified in Fig. 3. The numerical results with a space step $\delta_{x}=5 \cdot 10^{-2}$ obtained using the Rusanov scheme for the coupled model of shallow water equations, the Roe scheme for the uncoupled treatment [7] and the CPR scheme with two different time steps $\left(\delta_{t}=10^{-4}\right.$ and $\left.\delta_{t}=10^{-6}\right)$ are compared. The Godunov schemes require a time step $\delta_{t}=10^{-4}$ for stability while the CPR scheme can be used with a larger time step but the result is more diffusive. According to the asymptotic analysis, the time step has to be of the order of the potential wave celerity in order to approach the acoustic regime $\left(M_{0}^{\tau}\right)$.

The Godunov schemes are able to preserve the amplitude of the highest frequency, by adjusting the time step. However, the lower the frequencies are, the more diffusive the wave is and moreover the smaller the time step is, the larger the diffusion is. With the same time step used to ensure the stability of the Godunov schemes, i.e. $\delta_{t}=10^{-4}$, the CPR scheme preserves the amplitude of the lowest frequency wave. In addition, the smaller the time step is, the smaller the diffusion is for the high frequencies. Obviously, long-time simulations, from several hours to one year, are needed in the context of an oceanic flow, and the low frequencies are the most representative.

4.2. Lock exchange simulation. This test case is the simulation of the lock exchange experiment with the multilayer shallow water model. For more details about the test case and its physical interpretation, we refer to [20]. The initial condition is given by

$$
h_{2}^{0}(x)=\left\{\begin{array}{ll}
1.5 & \text {, if } x<0.5 \\
0.5 & \text {, elsewhere. }
\end{array}, \quad h_{1}^{0}(x)= \begin{cases}0.5 & \text {, if } x<0.5 \\
1.5 & \text {, elsewhere }\end{cases}\right.
$$

and $u_{1}^{0}=u_{2}^{0}=B=0$. In this configuration, the solution of the bilayer shallow water satisfies the hyperbolicity criterium highlighted in [31] for any well-stratified $\left(\varrho_{1}<\varrho_{2}\right)$ density ratio $\frac{\underline{\varrho}_{2}-\varrho_{1}}{\varrho_{2}}$. The simulations of this section are performed with a space step set to $\delta_{x}=5 \cdot 10^{-3}$. The reference solution is a converged numerical solution using a space step $\delta_{x}=10^{-5}$ and the CPR scheme. Godunov schemes converge to the same solution in the case of large density ratio. When the density ratio is small, Godunov schemes are so diffusive and the time step is so small that the converged solution is out of our reach.

4.2.1. Large density ratio. Fig. 4 shows the interface levels and the velocity profiles for a density ratio set to $\frac{\varrho_{2}-\varrho_{1}}{\varrho_{2}}=\frac{1}{2}$. The velocity resulting from the variation of density leads to a Froude number $\varepsilon=1$. The time step is estimated to satisfy the CFL condition (2.2) and leads to $\delta_{t} \approx 5 \cdot 10^{-4}$ for the regularization parameter $\gamma=1$. The classical CFL condition of Godunov schemes leads to the same time step. For the regularization parameter $\gamma=10$, the time step of the CPR scheme is slightly smaller $\delta_{t} \approx 10^{-4}$. The four waves of the Riemann problem corresponding to the initial condition can be clearly identified in Fig. 4. 


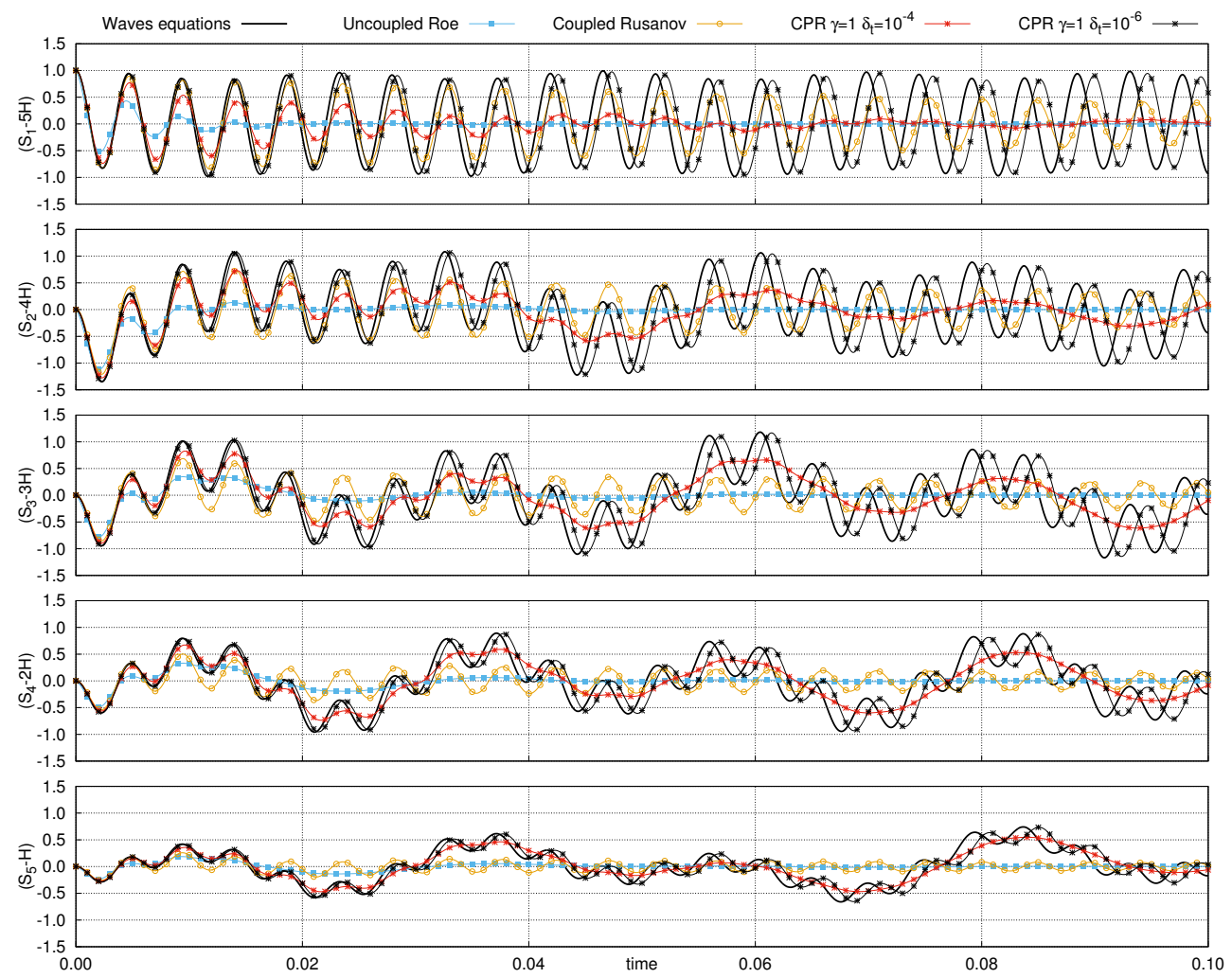

FIG. 3. Simulation of oscillating surfaces: Comparison between the CPR $\left(\gamma=1\right.$ with $\delta_{t}=10^{-4}$ and $\left.\delta_{t}=10^{-6}\right)$, classical Godunov schemes and the asymptotic acoustic regime $\left(M_{0}^{\tau}\right)$. Deviation of the interfaces $\left(S_{j}=\sum_{i=j}^{L} h_{i}\right)$ at the position $x=0$ along the time.

4.2.2. Small density ratio. Fig. 5 shows the interface levels and the velocity profiles for a small density variation, i.e. $\frac{\varrho_{2}-\varrho_{1}}{\varrho_{1}}=10^{-4}$. The velocity resulting from the variation of density leads to a Froude number $\varepsilon=10^{-3}$. The time step used for the Godunov solvers are almost the same $\delta_{t} \approx 5 \cdot 10^{-4}$. The Lax-Wendroff scheme require a smaller time step to give the results presented in Fig. 5 , i.e. $\delta_{t} \approx 10^{-4}$. A larger time step can be used with the CPR scheme and still satisfies the CFL condition (2.2), i.e. $\delta_{t} \approx 3 \cdot 10^{-3}$ for $\gamma=1$ and $\delta_{t} \approx 10^{-3}$ for $\gamma=10$. The numerical results Fig. 4 make two very different wave speeds appear. The barotropic mode is quite large $c_{1} \approx 4.5$ and the free surface is almost flat. On the contrary, at the interface, the variation of density create a very small current $c_{2} \approx 10^{-3}$. The solution observed is close to the solution of the incompressible regime $\left(M_{0}^{t}\right)$, the so-called rigid-lid in the shallow water context, i.e. $h_{1} u_{1}=-h_{2} u_{2}$.

5. Conclusion. A new numerical scheme for a system of advection equations with irrotational force term in multi-dimensional framework is proposed and analyzed. The numerical scheme is applicable to a large class of physical models. The strategy is particularly well adapted to multicomponent fluids and deals with the nonconservative interactions. The numerical strategy proposed does not require a bound of the eigenvalues of the system, which is not trivial for large system of equations. Several numerical illustrations are presented in the context of the multilayer shallow 

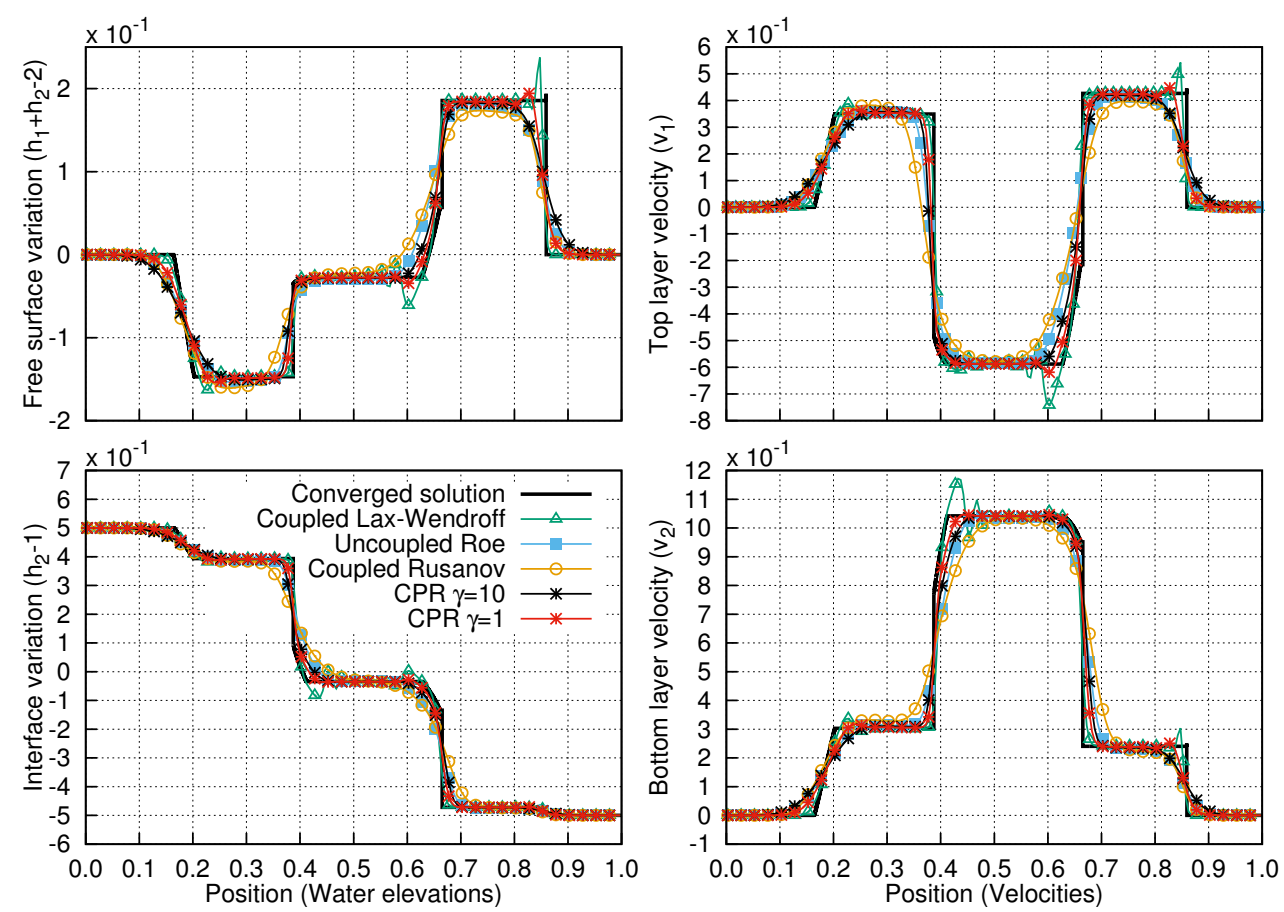

FIG. 4. Lock exchange simulation: Comparison between the CPR scheme $(\gamma=1$ and $\gamma=10)$ and Godunov schemes using coupled or uncoupled strategies with large density ratio $\left(\frac{\varrho_{2}-\varrho_{1}}{\varrho_{2}}=\frac{1}{2}\right)$ at time $t=0.08$ with $\delta_{x}=5 \cdot 10^{-3}$.

water model. The main advantages of the method is its strong robustness. It is still accurate in the limit where the forces are large compared with the advection terms. The dissipation of the mechanic energy, stand for a mathematical entropy, is proven even when a large advective time step is used. Numerical experiments show the presence of oscillations in the vicinity of shocks. The regularization parameter introduced in the scheme seems able to correct this drawback. However, the sensitivity of the solution to the regularization parameter is clearly depending on the test case and a fine parameter analysis is a perspective for further work. In a futur work, an extension of the method to the case where the potentials of the forces are not an explicit function of the mass will be proposed. It is in particular the case of the Euler system with internal energy equation [24, 29], the multi-polar Euler-Poisson equation [10] and the chemotaxis model [33].

Acknowledgements. This research is part of a collaborative program with the French Naval Hydrographic and Oceanographic Service (SHOM) through the grant 15CR02. The authors thank R. Baraille for many fruitful discussions and F. Couderc for his kind advice.

\section{REFERENCES}

[1] Audusse, E. A multilayer saint-venant model: derivation and numerical validation. Discrete Contin. Dyn. Syst. Ser. B 5 (2005), 189-214.

[2] Audusse, E., Bouchut, F., Bristeau, M.-O., Klein, R., and Perthame, B. A fast and stable well-balanced scheme with hydrostatic reconstruction for shallow water flows. SIAM 

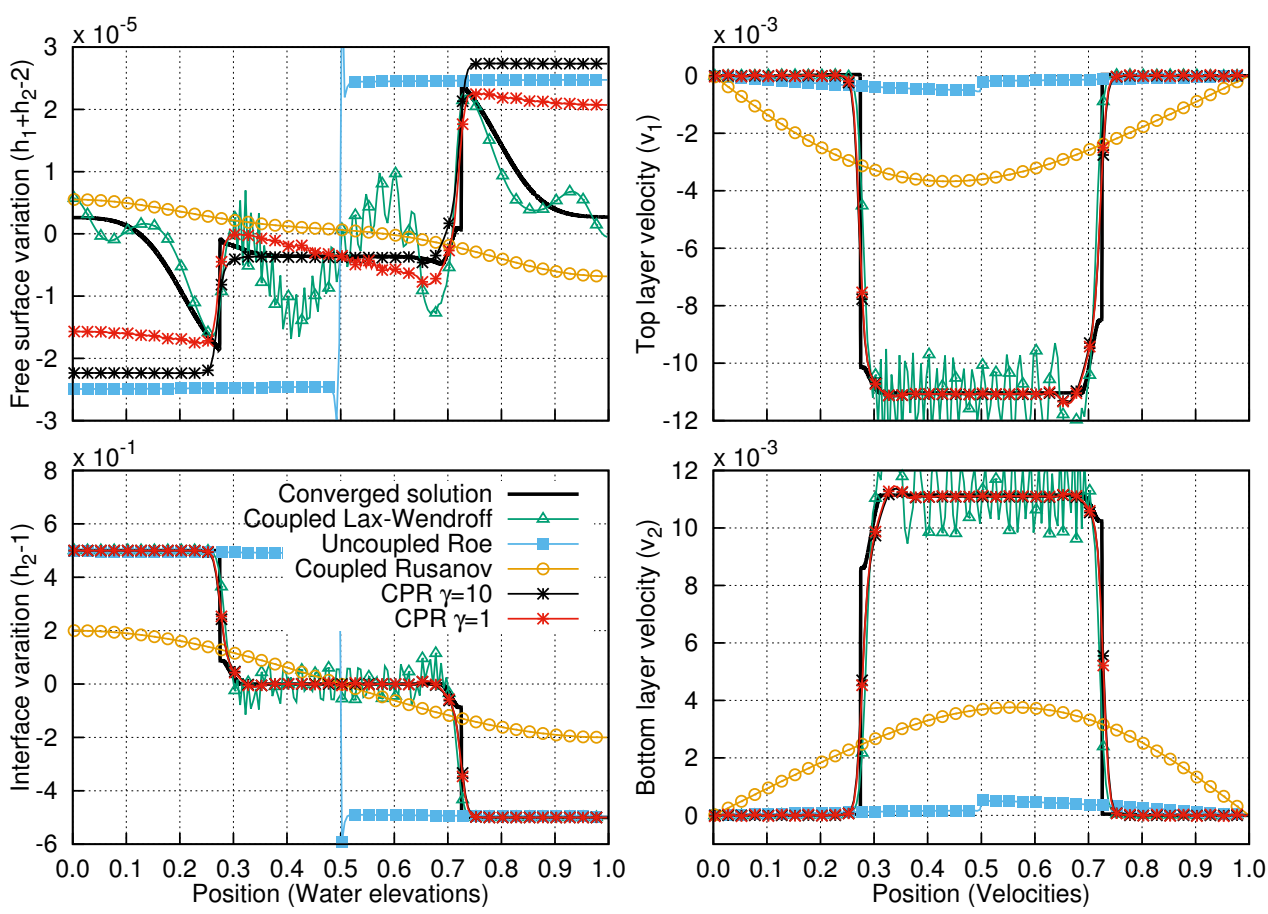

FIG. 5. Lock exchange simulation: Comparison between the $C P R\left(\gamma=1, \delta_{t}=10^{-3}\right)$ and Godunov schemes $\left(\delta_{t}=5 \cdot 10^{-4}\right)$ with small density variation $\left(\frac{\varrho_{2}-\varrho_{1}}{\varrho_{1}}=10^{-4}\right)$ at time $t=10$ with $\delta_{x}=5 \cdot 10^{-3}$.

J. Sci. Comput. 25, 6 (2004), 2050-2065.

[3] BedFord, A., And Drumheller, D. S. Theories of immiscible and structured mixtures. International Journal of Engineering Science 21, 8 (1983), 863-960.

[4] Bermudez, A., ANd Vazquez, M. E. Upwind methods for hyperbolic conservation laws with source terms. Comput. \& Fluids 23, 8 (1994), 1049-1071.

[5] Bernard, M., Dellacherie, S., Faccanoni, G., Grec, B., and Penel, Y. Study of a low mach nuclear core model for two-phase flows with phase transition I: stiffened gas law. ESAIM Math. Model. Numer. Anal. 48 (11 2014), 1639-1679.

[6] Bianchini, S. On the riemann problem for non-conservative hyperbolic systems. Arch. Ration. Mech. Anal. 166, 1 (2003), 1-26.

[7] Bouchut, F., AND DE LunA, T. M. An entropy satisfying scheme for two-layer shallow water equations with uncoupled treatment. ESAIM Math. Model. Numer. Anal. 42 (2008), 683698.

[8] Castro, M., Gallardo, J. M., López-García, J. A., and Parés, C. Well-balanced high order extensions of Godunov's method for semilinear balance laws. SIAM J. Numer. Anal. 46, 2 (2008), 1012-1039.

[9] Castro, M. J., Fernández-Nieto, E. D., Ferreiro, A. M., García-Rodríguez, J. A., And PARÉs, C. High order extensions of Roe schemes for two-dimensional nonconservative hyperbolic systems. J. Sci. Comput. 39, 1 (2009), 67-114.

[10] Chainais-Hillairet, C., Peng, Y.-J., AND Violet, I. Numerical solutions of Euler-Poisson systems for potential flows. Appl. Numer. Math. 59, 2 (2009), 301-315.

[11] De Saint-Venant, A.-J.-C. B. Théorie du mouvement non permanent des eaux, avec application aux crues des rivières et à l'introduction des marées dans leurs lits. $\underline{\text { C.R. Acad. Sci. }}$ Paris 73 (1871), 147-154.

[12] Dellacherie, S. Analysis of godunov type schemes applied to the compressible euler system at low mach number. J. Computational Phys. 229 (2010), 978-1016.

[13] Duchêne, V. Asymptotic shallow water models for internal waves in a two-fluid system with a free surface. SIAM J. Math. Anal. 42 (2010), 2229-2260. 
[14] Gangbo, W., and Westdickenberg, M. Optimal transport for the system of isentropic Euler equations. Comm. Partial Differential Equations 34, 7-9 (2009), 1041-1073.

[15] Gavrilyuk, S., AND Gouin, H. A new form of governing equations of fluids arising from hamilton's principle. Internat. J. Engrg. Sci. 37, 12 (1999), 1495-1520.

[16] Godlewski, E., AND Raviart, P.-A. Numerical approximation of hyperbolic systems of conservation laws, vol. 118 of Applied Mathematical Sciences. Springer-Verlag, New York, 1996.

[17] Greenberg, J. M., And Leroux, A.-Y. A well-balanced scheme for the numerical processing of source terms in hyperbolic equations. SIAM J. Numer. Anal. 33, 1 (1996), 1-16.

[18] Grenier, N., Vila, J.-P., And Villedieu, P. An accurate low-mach scheme for a compressible two-fluid model applied to free-surface flows. J. Computational Phys. 252 (2013), 1-19.

[19] Guillard, H., And Viozat, C. On the behaviour of upwind schemes in the low mach number limit. Computers \& Fluids 28, 1 (1 1999), 63-86.

[20] Ilcak, M., Adcroft, A. J., Griffies, S. M., and Hallberg, R. W. Spurious dianeutral mixing and the role of momentum closure. Ocean Modelling 45-46, 0 (2012), 37-58.

[21] JIN, S. Efficient asymptotic-preserving (ap) schemes for some multiscale kinetic equations. SIAM Journal on Scientific Computing 21, 2 (1999), 441-454.

[22] Jin, S., AND WEN, X. Two interface-type numerical methods for computing hyperbolic systems with geometrical source terms having concentrations. SIAM J. Sci. Comput. 26, 6 (2005), 2079-2101 (electronic).

[23] Klainerman, S., And Majda, A. Singular limits of quasilinear hyperbolic systems with large parameters and the incompressible limit of compressible fluids. Comm. Pure Appl. Math. 34, 4 (1981), 481-524.

[24] Klainerman, S., and Majda, A. Compressible and incompressible fluids. Communications on Pure and Applied Mathematics 35, 5 (1982), 629-651.

[25] Lax, P., And Wendroff, B. Systems of conservation laws, comm. Pure and Applied Maths 13 (1960), 217-237.

[26] Liou, M.-S., and Steffen JR, C. J. A new flux splitting scheme. J. Comput. Phys. 107, 1 (1993), 23-39.

[27] Liska, R., And Wendroff, B. Analysis and computation with stratified fluid models. J. Comput. Phys. 137, 1 (1997), 212-244.

[28] LonG, R. R. Long waves in a two-fluid system. Journal of Meteorology 13, 1 (1956), 70-74.

[29] Métivier, G., ANd Schochet, S. The incompressible limit of the non-isentropic euler equations. Arch. Ration. Mech. Anal. 158, 1 (2001), 61-90.

[30] Monjarret, R. Local well-posedness of the multi-layer shallow water model with free surface. To appear.

[31] Monjarret, R. Local well-posedness of the two-layer shallow water model with free surface. SIAM Journal on Applied Mathematics 75, 5 (2016/04/11 2015), 2311-2332.

[32] Muñoz-Ruiz, M. L., AND PARÉs, C. Some comments on the numerical approximation of hyperbolic nonconservative systems. In Numerical methods for hyperbolic equations: theory and applications. CRC Press/Balkema, Leiden, 2013, pp. 311-318.

[33] Natalini, R., Ribot, M., and Twarogowska, M. A well-balanced numerical scheme for a one dimensional quasilinear hyperbolic model of chemotaxis. Commun. Math. Sci. 12, 1 (2014), 13-39.

[34] Noelle, S., Pankratz, N., Puppo, G., and Natvig, J. R. Well-balanced finite volume schemes of arbitrary order of accuracy for shallow water flows. J. Comput. Phys. 213, 2 (2006), $474-499$.

[35] Noelle, S., Xing, Y., And Shu, C.-W. High-order well-balanced finite volume WENO schemes for shallow water equation with moving water. J. Comput. Phys. 226, 1 (2007), 29-58.

[36] Ovsyannikov, L. Two-layer "shallow water" model. J. Appl. Mech. Tech. Phys. 20, 2 (1979), $127-135$.

[37] Parisot, M., and Vila, J.-P. Numerical scheme for multilayer shallow-water model in the low-froude number regime. C.R. Acad. Sci. Paris, Ser. I 352 (2014), 953-957.

[38] Perthame, B., And Simeoni, C. A kinetic scheme for the saint-venant system with a source term. Calcolo 38, 4 (2001), 201-231.

[39] RannacheR, R. On Chorin's projection method for the incompressible Navier-Stokes equations. In The Navier-Stokes equations II-theory and numerical methods (Oberwolfach, 1991), vol. 1530 of Lecture Notes in Math. Springer, Berlin, 1992, pp. 167-183.

[40] Reznik, G., Zeitulin, V., and Ben Jelloul, M. Nonlinear theory of geostrophic adjustment. part 1. rotating shallow-water model. J. Fluid Mech. 445 (2001), 93-120.

[41] Serrin, J. Mathematical principles of classical fluid mechanics. In Handbuch der Physik (herausgegeben von S. Flügge), Bd. 8/1, Strömungsmechanik I (Mitherausgeber C. 
Truesdell). Springer-Verlag, Berlin-Göttingen-Heidelberg, 1959, pp. 125-263.

[42] Slemrod, M. Dynamic phase transitions in a van der waals fluid. J. Differential Equations 52, 1 (1984), 1-23.

[43] Strauss, W. A. Partial Differential Equations: An Introduction. John Wiley, 1992.

[44] Wijngaarden, L. V. On the equations of motion for mixtures of liquid and gas bubbles. J. Fluid Mech. 33, 03 (1968), 465-474. 